\title{
Faulty oxygen sensing disrupts angiomotin function in trophoblast cell migration and predisposes to preeclampsia
}

\author{
Abby Farrell, ${ }^{1,2}$ Sruthi Alahari, ${ }^{1,3}$ Leonardo Ermini, ${ }^{1}$ Andrea Tagliaferro, ${ }^{1}$ Michael Litvack, ${ }^{4}$ \\ Martin Post, ${ }^{2,4}$ and Isabella Caniggia $a^{1,2,3,5}$ \\ 'Lunenfeld-Tanenbaum Research Institute, Mount Sinai Hospital, Toronto, Ontario, Canada. Institute of Medical Sciences, \\ and ${ }^{3}$ Department of Physiology, University of Toronto, Toronto, Ontario, Canada. ${ }^{4}$ Program in Translational Medicine, Peter \\ Gilgan Centre for Research and Learning, Hospital for Sick Children, Toronto, Ontario, Canada. ${ }^{5}$ Department of Obstetrics \\ and Gynecology, University of Toronto, Toronto, Ontario, Canada.
}

Human placenta development and a successful pregnancy is incumbent upon precise oxygendependent control of trophoblast migration/invasion. Persistent low oxygen leading to failed trophoblast invasion promotes inadequate spiral artery remodeling, a characteristic of preeclampsia. Angiomotin (AMOT) is a multifaceted scaffolding protein involved in cell polarity and migration, yet its upstream regulation and significance in the human placenta remain unknown. Herein, we show that AMOT is primarily expressed in migratory extravillous trophoblast cells (EVTs) of the intermediate and distal anchoring column. Its expression increases after 10 weeks of gestation when oxygen tension rises and EVT migration/invasion peaks. Time-lapse imaging confirmed that the AMOT $80-\mathrm{kDa}$ isoform promotes migration of trophoblastic JEG3 and HTR8/SVneo cells. In preeclampsia, however, AMOT expression is decreased and its localization to migratory fetomaternal interface EVTs is disrupted. We demonstrate that Jumonji C domaincontaining protein 6 (JMJD6), an oxygen sensor, positively regulates AMOT via oxygen-dependent lysyl hydroxylation. Furthermore, in vitro and ex vivo studies show that transforming growth factor- $\beta$ (TCF- $\beta$ ) regulates AMOT expression, its interaction with polarity protein PAR6, and its subcellular redistribution from tight junctions to cytoskeleton. Our data reveal an oxygen- and TCF- $\beta$-driven migratory function for AMOT in the human placenta, and implicate its deficiency in impaired trophoblast migration that plagues preeclampsia.

Conflict of interest: The authors have declared that no conflict of interest exists.

Copyright: (C) 2019, American Society for Clinical Investigation.

Submitted: December 21, 2018 Accepted: March 14, 2019 Published: April 18, 2019

Reference information: /CI Insight. 2019;4(8):e127009. https://doi. org/10.1172/ji.insight.127009

\section{Introduction}

Angiomotin (AMOT), a multifunctional scaffolding protein originally discovered for its role in angiogenesis (1), has been shown to be localized at tight junctions, where it regulates apical-basolateral cell polarity in epithelial cells (2-5). Specifically, AMOT binds to components of the Par and Crumbs polarity complexes and sequesters them in the cytoplasm, leading to tight junction dissolution and changes in cell polarity (2). AMOT's function as a cell polarity regulator has been intricately linked to its role in cell migration. In endothelial cells, AMOT forms a ternary protein complex with polarity component Patj and RhoA GTPase exchange factor Syx at the leading edge of migrating cells (3). Furthermore, embryonic stem cells from Amot-deficient mice exhibited reduced cell migration rates due to cell polarization defects and failure of GTPase-activating protein Rich1 localization to lamellipodia (6). Recent studies have uncovered a novel role for AMOT as a modulator of early development, namely its role in cell-lineage specification of the inner cell mass and trophectoderm $(7,8)$. In the polarized trophectoderm, AMOT is found sequestered in the apical domain, thereby shielding its ability to bind to nuclear YAP and preventing transcription of genes involved in trophectoderm differentiation. Although this study reiterates how cell polarity and scaffolding, two features of AMOT, are essential for both embryonic and trophoectoderm cell differentiation, to our knowledge there has been no investigation into the role of AMOT in the human placenta.

During placenta development, connection of the placenta to the maternal decidua is imperative for establishment of the fetomaternal interface, and in turn a healthy pregnancy. This is achieved by differentiation of proliferative progenitor cytotrophoblasts $(\mathrm{CTs})$ into migratory/invasive extravillous trophoblast 
cells (EVTs), which infiltrate the maternal myometrium and transform the spiral arteries to achieve placental vascularization. Tightly controlled changes in oxygen tension and downstream canonical Smad-dependent and Smad-independent TGF- $\beta$ signaling experienced by the developing placenta have been demonstrated to regulate these events (9-11).

In preeclampsia (PE), a devastating pregnancy disorder affecting $5 \%-8 \%$ of pregnancies worldwide (12), EVTs are arrested in an immature, proliferative phenotype (13), and thereby exhibit shallow invasion into the maternal decidua $(14,15)$. Resultantly, insufficient spiral artery remodeling leads to reduced placental perfusion and persistent uteroplacental hypoxia (16). In fact, notable disruptions in upstream placental oxygen sensing machinery, including elevated hypoxia-inducible factor $1 \alpha(\mathrm{HIF}-1 \alpha)$, characterize early-onset PE (E-PE) (17). We have recently discovered a role for Jumonji C domain-containing protein 6 (JMJD6), a novel oxygen sensor belonging to a superfamily of JMJCD-containing proteins, in the human placenta $(18,19)$. JMJD6 was found to regulate the expression and stability of von Hippel-Lindau (VHL), a protein critical to the proteasomal degradation of HIF-1 $\alpha$ (18). However, in PE, persistence of low oxygen compromises JMJD6 enzymatic activity, and as a result, JMJD6-dependent lysyl hydroxylation and histone demethylation of VHL is impaired, leading to high HIF-1 $\alpha$ levels $(18,19)$. Studies have only recently started to uncover the novel substrates for JMJD6-mediated lysine hydroxylation, thereby implicating its role in distinct biological and pathological processes $(20,21)$. In the cancer field, JMJD6 has been found to exhibit oncogenic properties whereby it promotes the transcription of genes involved in cell proliferation (22), migration, and invasion (20,23).

This present study investigates AMOT in human placenta development and in PE. It implicates a function for AMOT in trophoblast cell migration, which is evidently impaired in PE. Further, it addresses how TGF- $\beta$ signaling, upstream changes in oxygen tension, and JMJD6 activity elicit control over AMOT functionality.

\section{Results}

Temporal and spatial expression of AMOT in the developing human placenta. We first characterized the spatial and temporal expression patterns of AMOT in the human placenta during early gestation. Expressed as 2 isoforms, the $80-\mathrm{kDa}$ product (AMOT 80 ) has been implicated in endothelial cell migration, whereas the $130-\mathrm{kDa}$ product (AMOT 130) is involved in changes in endothelial cell shape due to its distinguishing $\mathrm{N}$-terminal extension that promotes its association with the F-actin cytoskeleton $(24,25)$. Protein analysis revealed that both AMOT 130 and 80 isoforms are expressed in the human placenta as early as 5 weeks of gestation, and their expression significantly increased after 10 weeks (Figure 1A), a critical time point when changes in oxygen tension and TGF- $\beta$ signaling are known to occur $(10,26)$. Quantitative PCR analysis revealed that $A M O T$ mRNA expression is higher in placentae from 10 to 15 weeks of gestation, compared with placentae from 5 to 9 weeks of gestation (Figure 1B). These analyses were performed on whole placenta samples, thus encompassing a heterogenous mixture of trophoblasts. Analysis of AMOT expression in distinct trophoblast subpopulations isolated through laser capture microdissection (LCM) (27) demonstrated AMOT expression in syncytiotrophoblasts (STs) and CTs and proximal (PC) and distal column (DC) trophoblasts (Figure 1C). However, with advancing gestation, AMOT expression only increased in the ST/ CT layer, where trophoblast cells are undergoing active fusion, and more importantly in the DC, where migratory and invasive EVTs reside (Figure 1C). This was corroborated by immunohistochemical analysis of AMOT in first-trimester placentae sections, which revealed (a) a striking localization of AMOT to the cell boundaries of EVTs comprising the anchoring column, particularly restricted to the intermediate and distal regions of the EVT column and absent in the proximal region; and (b) AMOT localization to the underlying, proliferative CTs, as well as in the overlying, multinucleated ST layer with advancing gestation (Figure 1D). During placenta development, critical cellular events, including trophoblast migration, rely on tightly regulated changes in oxygen tension. Hence, we examined the effect of low oxygen on AMOT expression levels. Exposure of trophoblast-derived JEG3 cells to 3\% oxygen significantly decreased AMOT 130 and 80 protein levels compared with normoxic $21 \%$ oxygen (Figure $1 \mathrm{E}$ ).

TGF- $\beta$ regulates AMOT expression, subcellular localization, and interaction with PAR6. During the early events of trophoblast differentiation, low oxygen tension via HIF-1 $\alpha$ has been demonstrated to upregulate levels of TGF- $\beta 3$ (10). Further, we have shown a TGF- $\beta$-dependent regulation of polarity protein PAR6 in guiding trophoblast cell migration (28). Considering that AMOT is a scaffolding protein implicated in cell polarity, we next investigated if there was a TGF- $\beta$-dependent regulation of AMOT utilizing JEG3 cells. Treatment of JEG3 cells with $10 \mathrm{ng} / \mathrm{ml}$ TGF- $\beta 1 / 3$ ligand for 24 hours resulted in a significant 
A

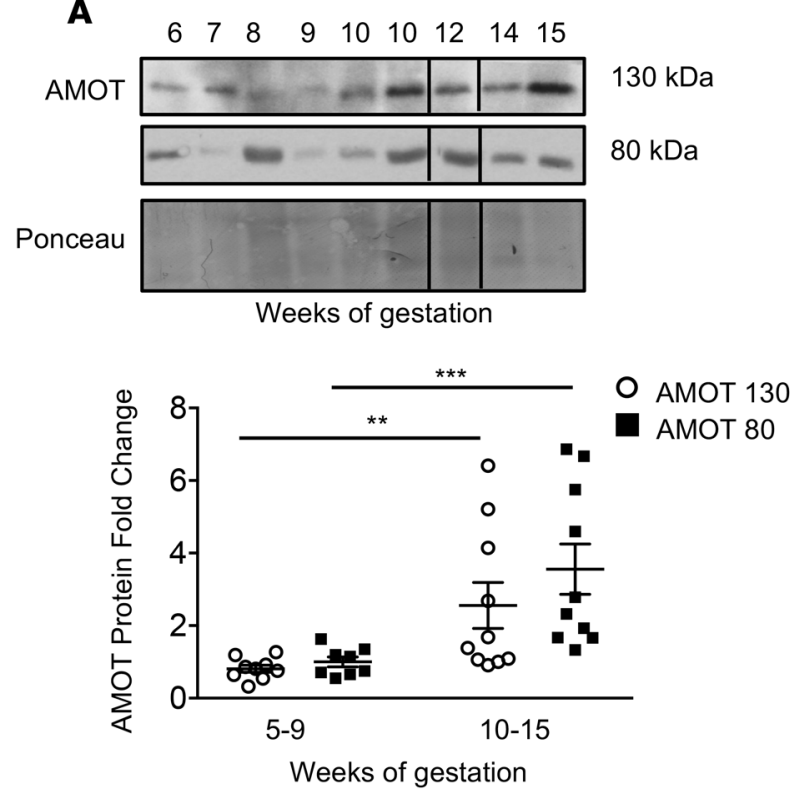

B

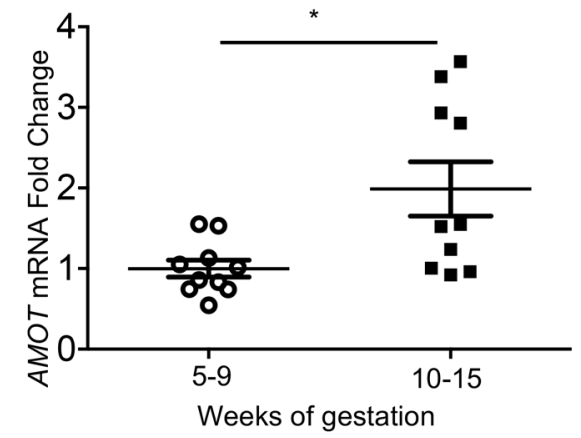

C

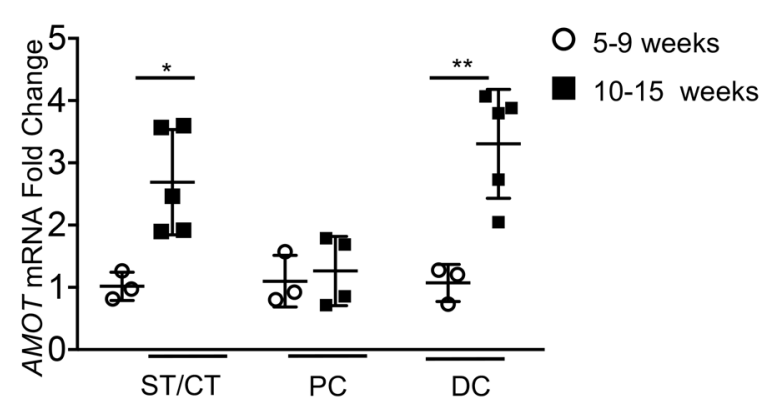

D

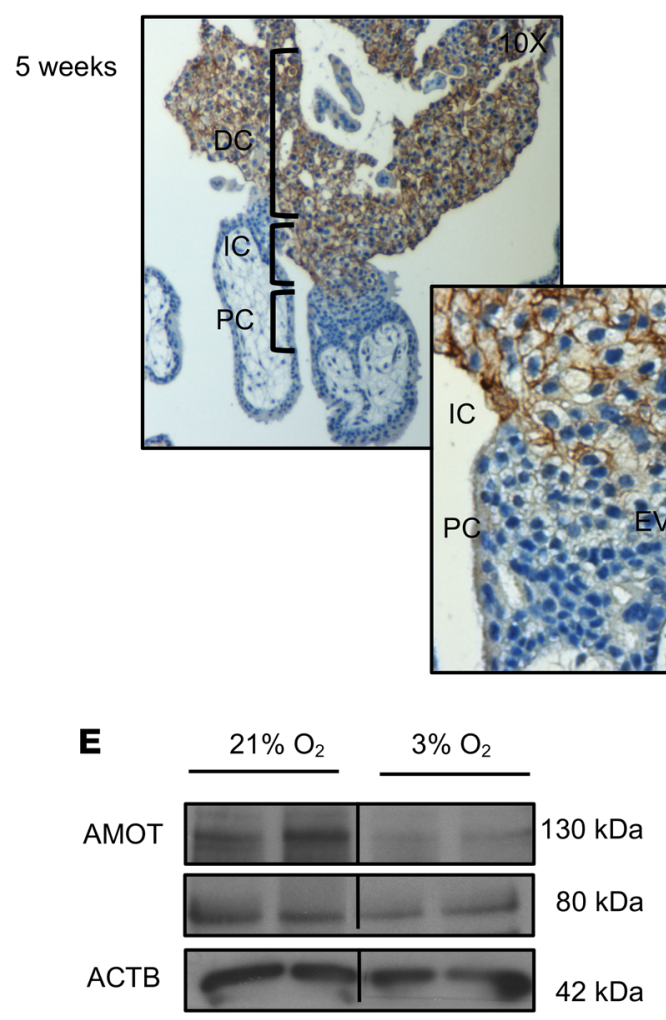

13 weeks
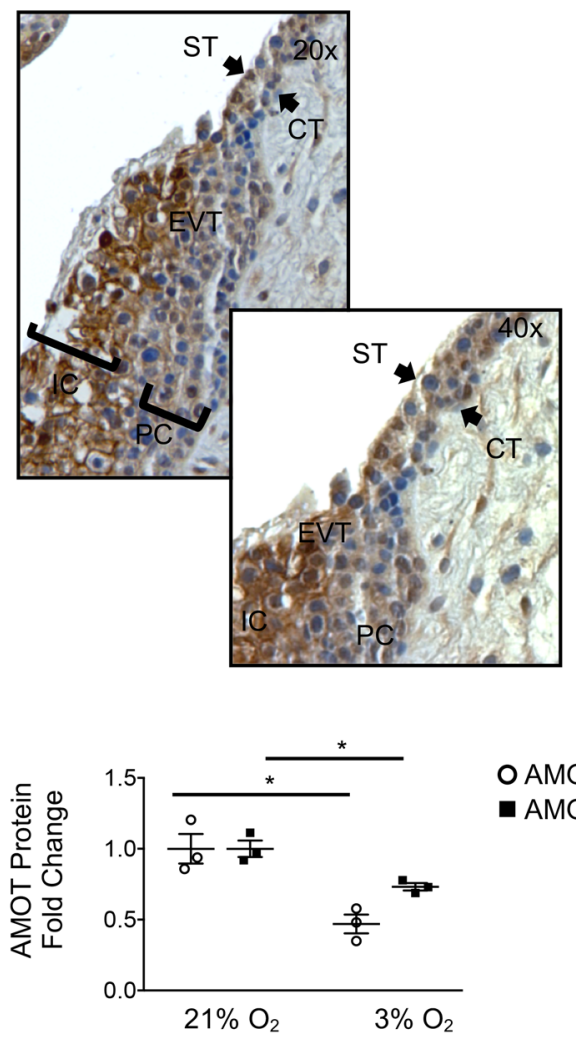

O AMOT 130

- AMOT 80

Figure 1. Temporal and spatial expression of AMOT in early placenta development. (A) Representative Western blot (WB) of AMOT and associated densitometry in human placenta lysates from 5 to 15 weeks of gestation. AMOT protein levels were normalized by Ponceau staining and expressed as fold change relative to $5-9$ weeks. ${ }^{* *} P<0.01,{ }^{* *} P<0.001$ by nonparametric Mann-Whitney $U$ test $(5-9$ weeks, $n=9 ; 10-15$ weeks, $n=10)$. (B) qPCR for AMOT in human placenta from 5 to 15 weeks of gestation. Data are expressed as fold change relative to $5-9$ weeks. ${ }^{*} P<0.05$ by nonparametric Mann-Whitney $U$ test (5-9 weeks, $n=10 ; 10-15$ weeks, $n=10)$. (C) qPCR for AMOT in villous syncytiotrophoblast/cytotrophoblast (ST/CT) layer, and extravillous proximal column (PC) and distal column (DC) in first-trimester placental sections obtained via laser capture microdissection. ${ }^{*} P<0.05$, ${ }^{*} P<0.01$ by 2-tailed unpaired Student's $t$ test (5-9 weeks, $n=3 ; 10-15$ weeks, $n=4$ or 5). (D) Representative images of IHC staining of AMOT in sections of human placenta from 5 to 6 weeks versus 10 to 12 weeks of gestation ( $5-6$ weeks, $n=7 ; 10-12$ weeks, $n=4$ ). Arrows indicate AMOT localization to specific cell types and structures within the placenta (DC, distal column; IC, intermediate column; PC, proximal column; ST, 
syncytiotrophoblast; CT, cytotrophoblast; EVT, extravillous trophoblast). Original magnification, $\times 10$ and $\times 40$ (left panel) and $\times 20$ and $\times 40$ (right panel). (E) Representative WB of AMOT and associated densitometry in JEG3 cells following exposure to $21 \%$ or $3 \%$ oxygen for 24 hours. AMOT protein levels were normalized to $\beta$-actin (ACTB) and expressed as fold change relative to cells maintained at $21 \%$ oxygen. In $\mathbf{A}$ and $\mathbf{E}$, lanes were run on the same gel but were noncontiguous. ${ }^{*} P<0.05$ by 2 -tailed unpaired Student's $t$ test $(n=3)$.

decrease in AMOT content compared with vehicle controls (Figure 2A), uncovering TGF- $\beta$ as a negative regulator of AMOT protein. Activation of TGF- $\beta$ signaling was confirmed by an increase in p-SMAD2 levels. Dual-staining immunofluorescence (IF) displayed a distinct AMOT colocalization with tight junction marker ZO-1 at intracellular tight junctions, and AMOT localization to leading edges of JEG3 cells (Figure 2B). Quantification of AMOT and ZO-1 colocalization by Pearson's correlation confirmed strong association in unstimulated culture conditions (Pearson's correlation coefficient $[\mathrm{PCC}]=0.56$ ) (Figure 2C). However, following TGF- $\beta 1 / 3$ treatment, AMOT localization at tight junctions is intermittent and disrupted compared with vehicle controls, as confirmed by a reduction in Pearson's correlation of AMOT and ZO-1 (PCC $=0.25$ and PCC $=0.27$, respectively) (Figure 2C). Intriguingly, TGF- $\beta 1 / 3$ treatment resulted in redistribution of AMOT to the cytoplasm in a fiber-like orientation. IF analysis of AMOT and the F-actin marker phalloidin following TGF- $\beta 1 / 3$ treatment underscored that TGF- $\beta$ promoted AMOT redistribution from the tight junction to the F-actin cytoskeleton (Figure 2D). Quantification confirmed increased AMOT and phalloidin colocalization following TGF- $\beta 1$ and $-\beta 3$ treatment $(\mathrm{PCC}=0.65$ and PCC $=0.58$, respectively) compared with vehicle control $(\mathrm{PCC}=0.21)$. To establish whether TGF- $\beta$ regulates AMOT in an ex vivo model, we analyzed AMOT in 5- to 9-week villous explants kept at $3 \% \mathrm{O}_{2}$ treated with antisense oligonucleotides against TGF- $\beta 3$ (AS-TGF- $\beta 3$ ); placental villi are inherently a major source of TGF- $\beta$ and thus inhibition of TGF- $\beta$ is optimal to examine downstream functional consequences (29). IF analysis revealed AMOT expression to be higher in explants treated with AS-TGF- $\beta 3$ compared with sense-treated controls (Figure 2E). Further, inhibition of TGF- $\beta 3$ maintained AMOT localization to distal mobile EVTs, as indicated by the positive human leukocyte antigen G (HLA-G) staining of these cells (Figure 2E). Low oxygen via HIF- $1 \alpha$ is an upstream positive regulator of TGF- $\beta 3$ (10). Accordingly, we observed similar results in explants kept at $3 \% \mathrm{O}_{2}$ treated with AS-HIF-1 $\alpha$ where AMOT localization to HLA-G-positive cells was increased compared with controls (Supplemental Figure 1A; supplemental material available online with this article; https://doi.org/10.1172/jci.insight.127009DS1). These findings confirm that oxygen and TGF- $\beta$ also regulate AMOT levels and tight junction localization in native trophoblast cells. Treatment of JEG3 cells with TGF- $\beta 1 / 3$ in the presence of a small-molecular inhibitor (SB-431542) of the ALK5 receptor inhibited Smad2 phosphorylation while retaining AMOT at cell boundaries and preventing cytoplasmic AMOT redistribution (Supplemental Figure 1B). These data confirm that the TGF- $\beta$-mediated localization of AMOT to the cytoskeleton is regulated by the Smad-dependent pathway.

Tankyrases (proteins involved in the poly-ADP-ribosylation of substrates) target AMOT for degradation via E3 ligase RNF146; therefore, inhibition of tankyrase activity stabilizes AMOT family proteins by preventing their degradation (30). In HTR-8/SVneo cells, treatment with tankyrase inhibitor XAV-939 increased protein levels of AMOT in a dose-dependent manner (Supplemental Figure 2A). Having established a negative regulation of AMOT by low oxygen, we next investigated whether this could be reversed through treatment with tankyrase inhibitor XAV-939. Interestingly, AMOT downregulation seen following exposure to low oxygen did not occur in the presence of XAV-939 (Supplemental Figure 2B). This was corroborated in IF analyses, which demonstrate that the AMOT fluorescence, and its localization to tight junctions, is maintained at the tight junctions of JEG3 cells in the presence of XAV939 in JEG3 cells cultured at $3 \%$ oxygen (Supplemental Figure 2C). These findings suggest a mechanism of low-oxygen-mediated downregulation of AMOT that involves an interplay with tankyrase-targeted degradation.

Under regulation by Smad-independent TGF- $\beta$ signaling, PAR6 functions to disrupt apical-basolateral cell polarity (31). In JEG3 cells, TGF- $\beta$ signaling via PAR6 was found to be active, where it specifically was shown to regulate trophoblast cell fusion and migration $(28,32)$. However, the interplay between PAR6 and AMOT, and further the role of TGF- $\beta$ signaling on this potential interaction, remains to be established. IF analysis revealed a colocalization of AMOT and PAR6 in the cytoplasm and at cell boundaries, which is further upregulated following TGF- $\beta 1 / 3$ exposure (Supplemental Figure 1D). IP of PAR6 followed by Western blot (WB) analysis of AMOT confirmed an AMOT and PAR6 interaction that is upregulated, particularly following TGF- $\beta 3$ treatment (Supplemental Figure 1C). Interestingly, AMOT/PAR6 interaction was only observed with the AMOT 80 isoform (Supplemental Figure 1C). This interaction between 
A

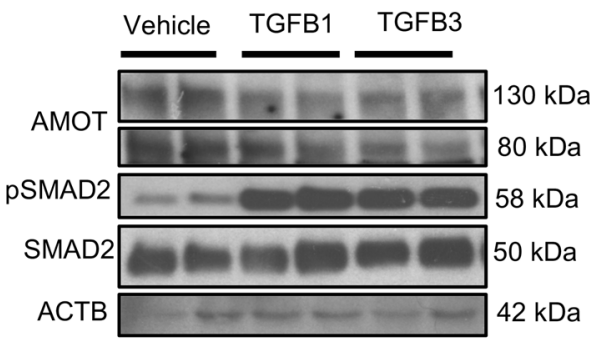

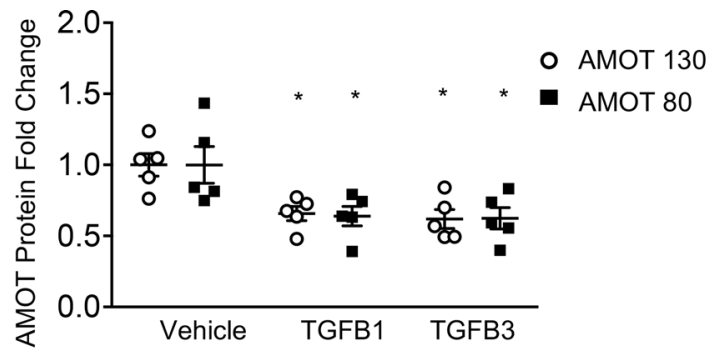

B

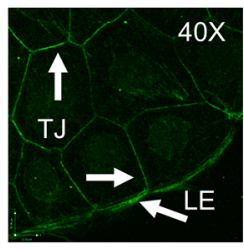

AMOT

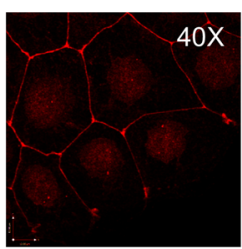

ZO-1

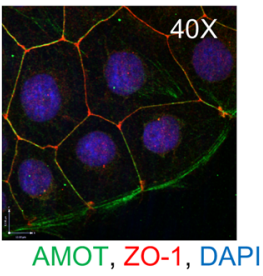

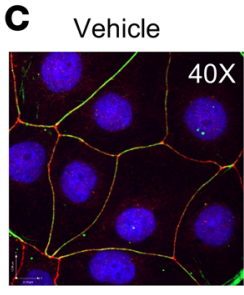

$\lg G$

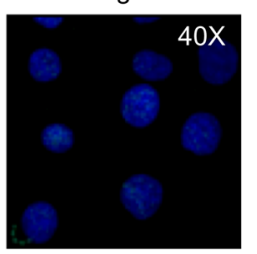

AMOT ZO-1 DAP
TGFB1

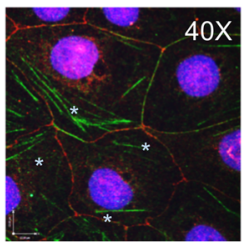

TGFB3

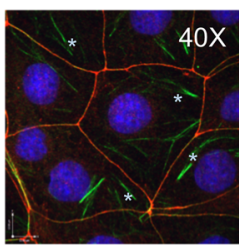

E

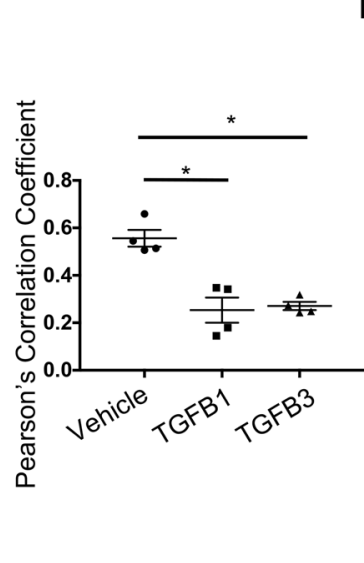

D Vehicle

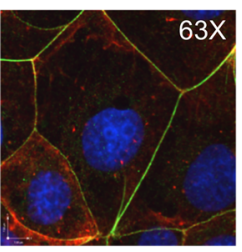

$\lg G$

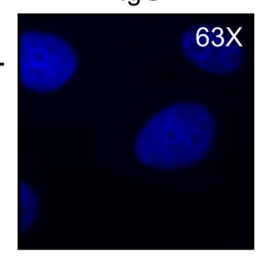

AMOT Phalloidin DAP
TGFB1

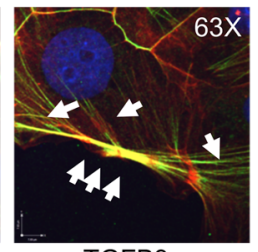

TGFB3

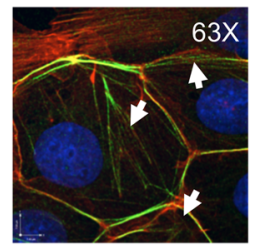

$63 x$

\section{x}

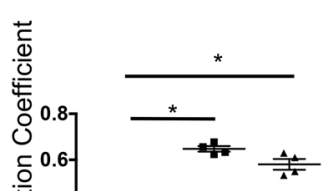

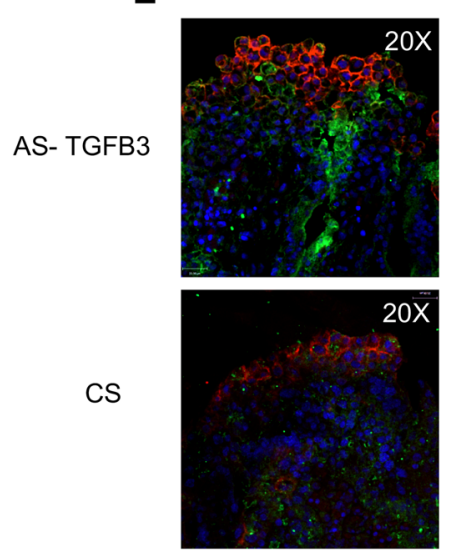

AMOT HLA-G DAPI

\section{villous explants}
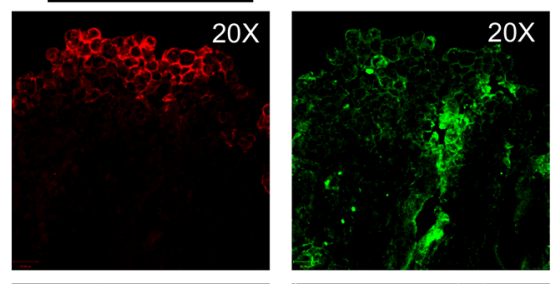

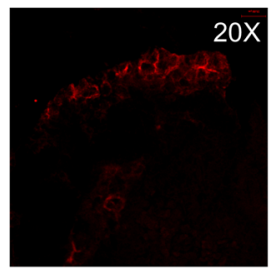

HLA-G

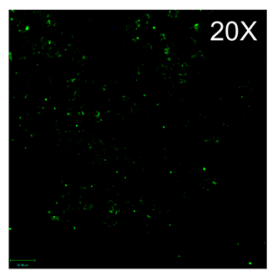

AMOT

Figure 2. AMOT levels and cellular localization are regulated by TGF- $\beta$ signaling. (A) Representative Western blot (WB) of AMOT and associated densitometry, and WB of p-SMAD2 and total SMAD2, following treatment with $10 \mathrm{ng} / \mathrm{ml}$ TCF- $\beta 1$ (TCFB1) or TCF- $\beta 3$ (TCFB3) for 24 hours. AMOT protein levels were normalized to $\beta$-actin (ACTB) and expressed as fold change relative to vehicle control. ${ }^{*} P<0.05$ by nonparametric Mann-Whitney $U$ test $(n=5$ separate experiments run in duplicate). (B) Representative immunofluorescence (IF) images of AMOT (green) and ZO-1 (red) in JEG3 cells cultured in standard conditions. Arrows indicate AMOT localization to tight junction (TJ) and leading edge (LE) ( $n=3$ individual experiments). Original magnification, $x 40$. (C) Representative IF images and colocalization analysis of AMOT (green) and ZO-1 (red) following treatment with $10 \mathrm{ng} / \mathrm{ml}$ TCF- $\beta 1$ or TCF- $\beta 3$ for 24 hours (Pearson's correlation coefficient $[P C C]$ : vehicle $=0.56$, TCF- $\beta 1=0.25$, TCF- $\beta 3=0.27$ ). Asterisks indicate AMOT redistribution to cytoplasm in fiber-like orientation. Original magnification, $x 40$. (D) Representative IF images and colocalization analysis of AMOT (green) and phalloidin (red) following treatment with $10 \mathrm{ng} / \mathrm{ml}$ TCF- $\beta 1$ or TCF- $\beta 3$ for 24 hours (PCC: vehicle $=0.21$, TCF- $\beta 1=0.65$, TCF- $\beta 3=0.58$ ). Arrows indicate AMOT colocalization to F-actin fibers. Original magnification, $\times 63$. Anti-goat IgC was used as control and nuclei were stained with DAPI (blue). ${ }^{*} P<0.05$ by nonparametric Mann-Whitney $U$ test $(n=4)$ for both $\mathbf{C}$ and $\mathbf{D}$. (E) Representative IF images of AMOT (green) and HLA-C (red) in villous explants at 5 to 8 weeks of gestation treated with $10 \mu \mathrm{M}$ control sense (CS) or antisense oligonucleotides against TGF- $\beta 3$ (AS-TCFB3). Explants were cultured in 3\% oxygen ( $n=3$ independent experiments). Original magnification, $\times 20$. 
AMOT and PAR6, and its regulation by TGF- $\beta$, was confirmed using proximity ligation assay (PLA), which showed (a) an AMOT and PAR6 association, as indicated by the presence of individual red PLA fluorophores (identified as dots); and (b) AMOT/PAR6 interaction significantly increased in TGF- $\beta 1 / 3-$ treated cells compared with vehicle control (Figure 3A).

PDZ-binding domain on AMOT is important for its interaction with PAR6. PDZ-binding domains are known interaction motifs found on various polarity proteins and tight junction-residing proteins (33). Notably, both AMOT and PAR6 contain this putative PDZ-binding domain (34). Considering this, the importance of the PDZ on AMOT in the interaction with PAR6 was investigated. Co-overexpression of PAR6 and one of the validated AMOT constructs (Figure 3B) demonstrated that overexpression of AMOT 80, but not AMOT 130, increases AMOT/PAR6 association compared with empty vector controls as assessed by WB (Figure 3B). Furthermore, overexpression of AMOT 80 with a mutated PDZ-binding domain (delta PDZ) decreased the association of AMOT 80 and PAR6 compared with overexpression of WT AMOT 80 (Figure 3B). PLA analysis confirmed the increase in AMOT/PAR6 interaction following overexpression of AMOT 80 compared with empty vector controls and highlighted the importance of the PDZ-binding domain for AMOT 80/PAR6 interaction to occur, as the AMOT 80 construct containing a mutated PDZ-binding domain led to an abrogated interaction (Figure 3C). Taken together, these data suggest that PAR6 primarily interacts with AMOT 80, and that the PDZ-binding motif contained in AMOT 80 is critical for this interaction. Considering that degradation of GTPase protein RhoA and dissolution of tight junctions is a consequence of active PAR6/SMURF signaling and precedes cell migration $(35,36)$, the contribution of AMOT 80 to tight junction stability and RhoA was analyzed. Overexpression of AMOT 80 resulted in a significant decrease in RhoA protein levels compared with empty vector controls (Figure 4D), indicating that the AMOT 80 interplay with PAR6 destabilizes tight junctions in JEG3 cells.

AMOT levels are reduced and localization to EVTs is impaired in PE. Reports from our lab have demonstrated that increased TGF- $\beta$ signaling because of chronic low oxygen contributes to the impaired trophoblast migration/invasion seen in PE $(10,29)$. Hence, we next investigated AMOT in E-PE placentae. WB demonstrated a significant decrease in AMOT 130 and AMOT 80 protein levels in E-PE compared with preterm control (PTC) placentae (Figure 4A). Although placentae delivered at a mean gestational age of 30 weeks are not "normal" relative to placentae delivered at term, AMOT protein levels were not different between placentae from preterm and term deliveries (data not shown), underscoring the utility of PTC for this study. Appreciating the deficiencies in trophoblast migration and invasion that typify PE, we next assessed AMOT localization to EVTs located at the maternal-fetal interface, particularly where interstitial EVTs have invaded and resided in the maternal decidua. In PTC and term control placentae, AMOT is found localized to the interstitial EVTs (Figure 4B), while in E-PE, AMOT localization to interstitial EVTs is markedly reduced. Positive HLA-G fluorescence within these cells confirms that they are interstitial EVTs. We next questioned if the interaction of AMOT 80 and PAR6 would also be disrupted. Analysis of AMOT 80 protein levels following IP of PAR6 in PTC and E-PE placenta lysates revealed a reduced AMOT 80 and PAR6 association in E-PE placentae (Figure 4C). To model the hypoxic milieu of PE, we next exposed HTR-8/SVneo cells, an established trophoblast cell line that exhibit EVT-like properties, to 3\% oxygen and standard $21 \%$ normoxic conditions. PLA analysis of AMOT and PAR6 interaction revealed that HTR-8/SVneo cells cultured in 3\% oxygen exhibited a significantly reduced AMOT and PAR6 interaction compared with cells cultured in $21 \%$ oxygen. These data suggest that the hypoxic environment characterizing $\mathrm{PE}$ contributes to impaired AMOT 80/PAR6 interaction.

AMOT 80 promotes trophoblast cell migration. Considering AMOT localization to migratory EVTs (Figures $1 \mathrm{D}$ and $4 \mathrm{~B}$ ), changes in AMOT distribution during cell migration were assessed via wound healing assay. Immediately following wound initiation in JEG3 cells, AMOT is primarily localized to the tight junctions (Figure 5A), consistent with previous observations of endogenous AMOT localization in resting cells (Figure 2B). However, following wound initiation, AMOT distinctly appeared in a fiber-like orientation at the leading edge of migrating cells, and in the cytoplasm of cells directly underlying the migratory front (Figure 5A), indicating that AMOT localization is altered during cell migration. To fully grasp the extent of AMOT spatial distribution in real time, we performed time-lapse imaging in JEG3 cells following wound healing assay. Visualization of AMOT in live cells was permitted through the use of a YFP-labeled AMOT 80 construct (Figure 5B), the dominant AMOT isoform involved in cell migration (3). During cell migration, AMOT 80 localization was observed to be in constant flux 
A

Negative Control

minus probe absent

Vehicle

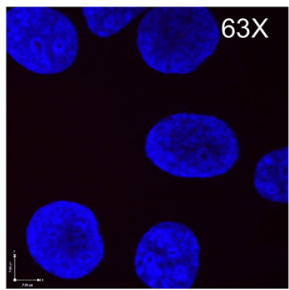

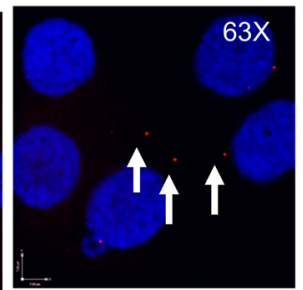

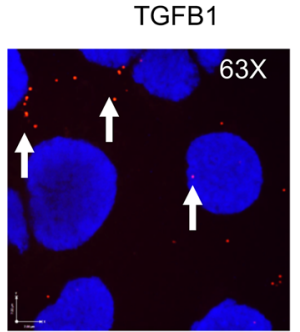

PLA DAPI

TGFB3

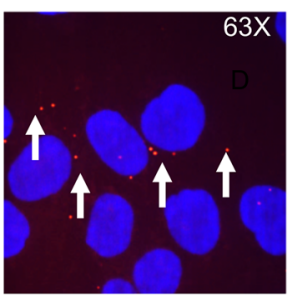

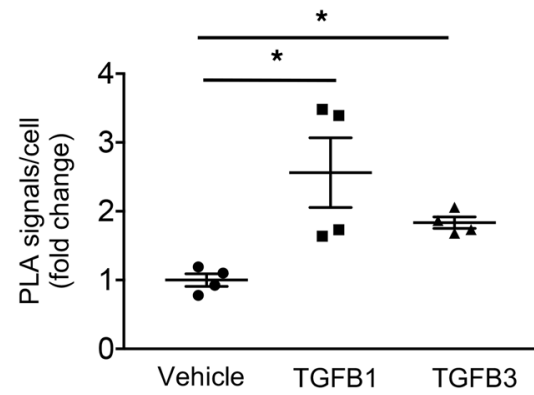

B
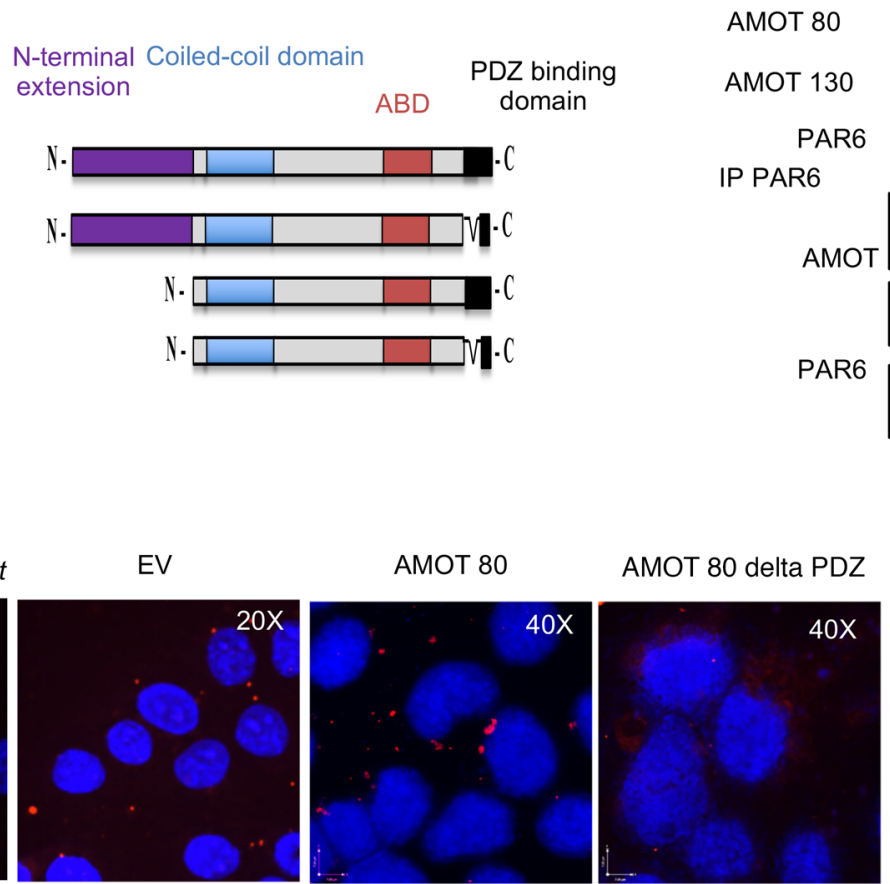

AMOT 130

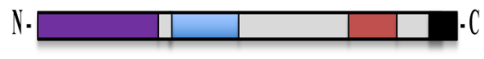

AMOT 130 delta PDZ

AMOT 80

AMOT 80 delta PDZ

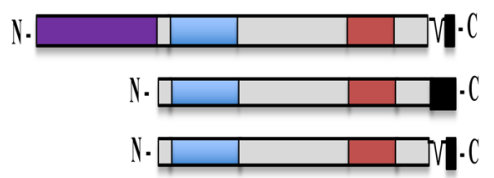

EV

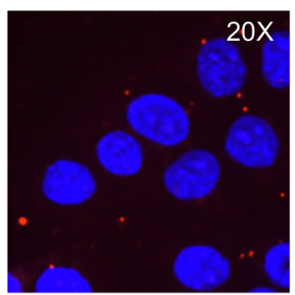

AMOT 80

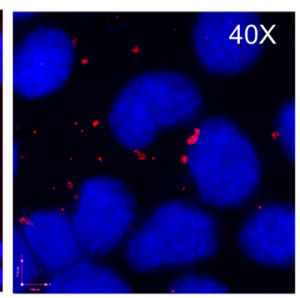

PLA DAPI
AMOT 80 delta PDZ

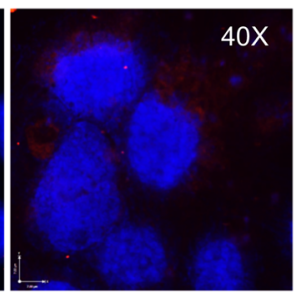

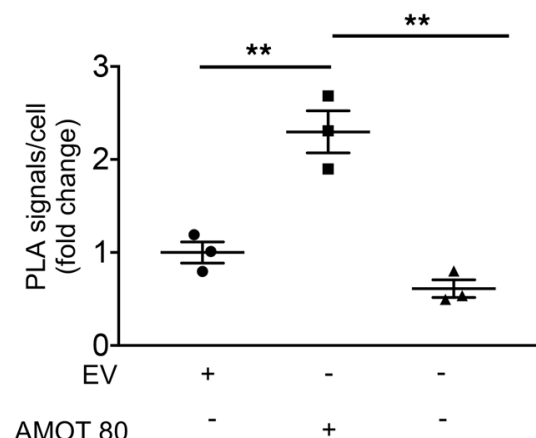

AMOT 80 delta PDZ

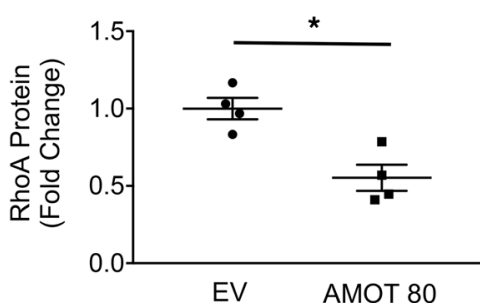

Figure 3. AMOT interacts with PAR6 under the regulation of TGF- $\boldsymbol{\beta}$. (A) Representative images depicting in situ proximity ligation assay (PLA) of AMOT with PAR6 in JEG3 cells following treatment with TCF- $\beta 1$, TGF- $\beta 3$, or vehicle control. Negative control was PLA reaction missing the minus (-) PLA probe and nuclei were stained with DAPI (blue). Original magnification, $\times 63$. Data were quantified as number of PLA signals per nucleus (cell) and expressed as fold change relative to vehicle control. ${ }^{*} P<0.05$ by nonparametric Mann-Whitney $U$ test $(n=4)$. (B) IP of PAR6 followed by Western blot (WB) of AMOT in JEG3 cells after co-overexpression of PARD6 with AMOT 130, AMOT 80, AMOT 130 delta PDZ, or AMOT 80 delta PDZ ( $n=3$ ). (C) Representative images depicting in situ PLA of AMOT with PAR6 in JEG3 cells following overexpression of empty vector (EV), AMOT 80, or AMOT 80delta PDZ. Negative control was PLA reaction missing the minus (-) PLA probe. Nuclei were stained with DAPI (blue). Original magnification, $\times 40$ and $\times 20$ (for EV). Data were quantified as number of PLA signals per nucleus (cell) and expressed as fold change relative to EV control. ${ }^{* *} P<0.01$ by 2 -tailed unpaired Student's $t$ test $(n=3)$. (D) Representative WB of RhoA and AMOT 80 protein levels following overexpression of EV or AMOT 80 in JEG3 cells (lanes were run on the same gel but were noncontiguous). Densitometric analysis of AMOT protein levels, which were normalized to $\beta$-actin (ACTB) and expressed as fold change relative to EV control. ${ }^{*} P<0.05$ by unpaired Mann-Whitney $U$ test $(n=4$ separate experiments). 


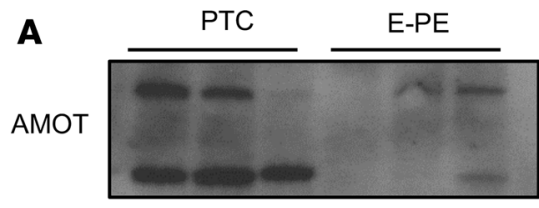

Ponceau

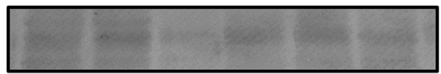

B

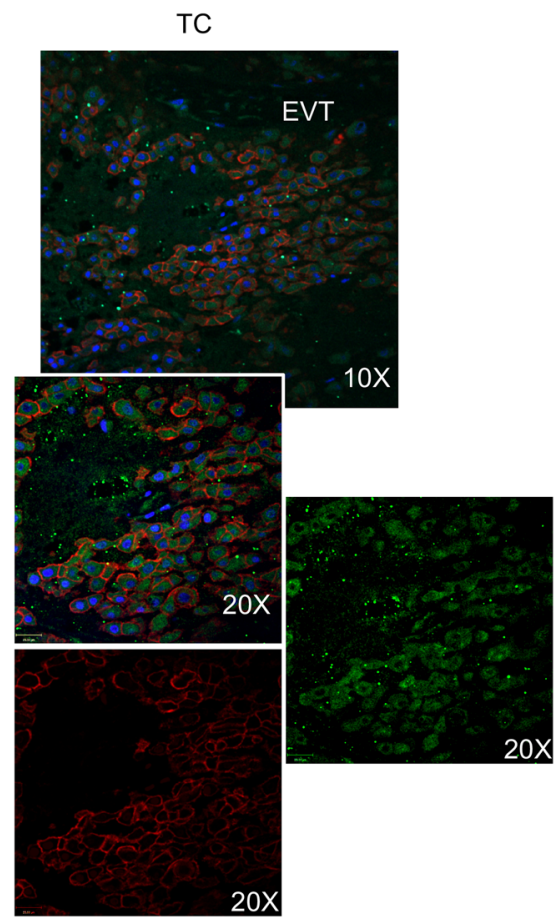

AMOT HLA-G DAPI
$130 \mathrm{kDa}$

$80 \mathrm{kDa}$

\section{Feto-maternal interface}

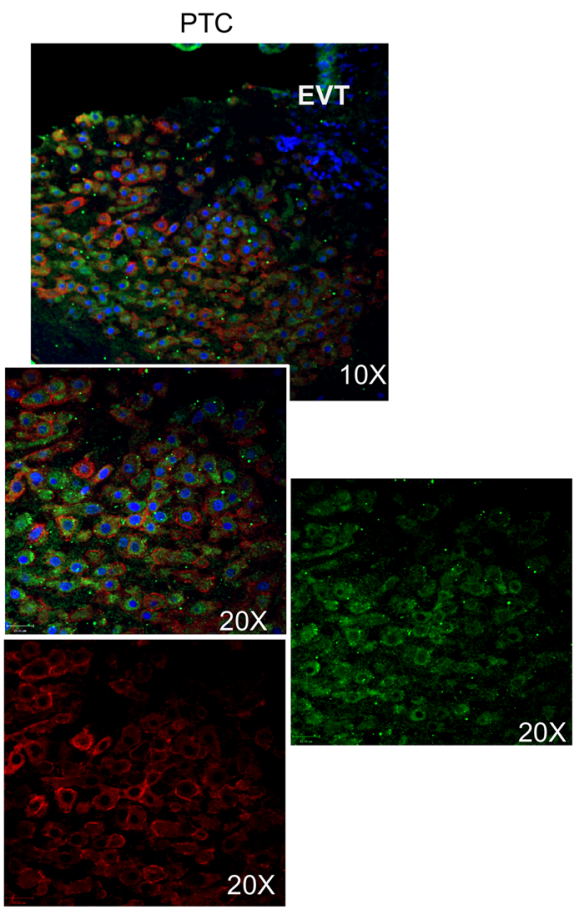

AMOT HLA-G DAPI

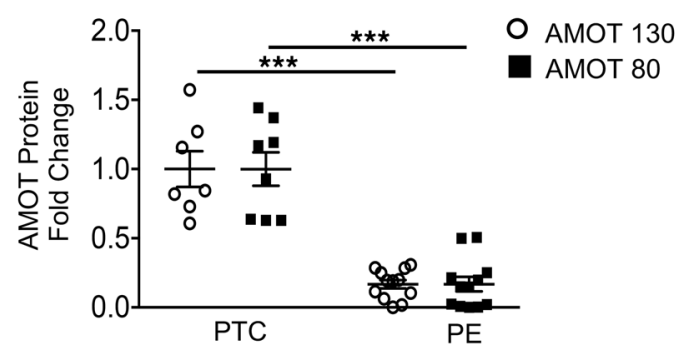

PE

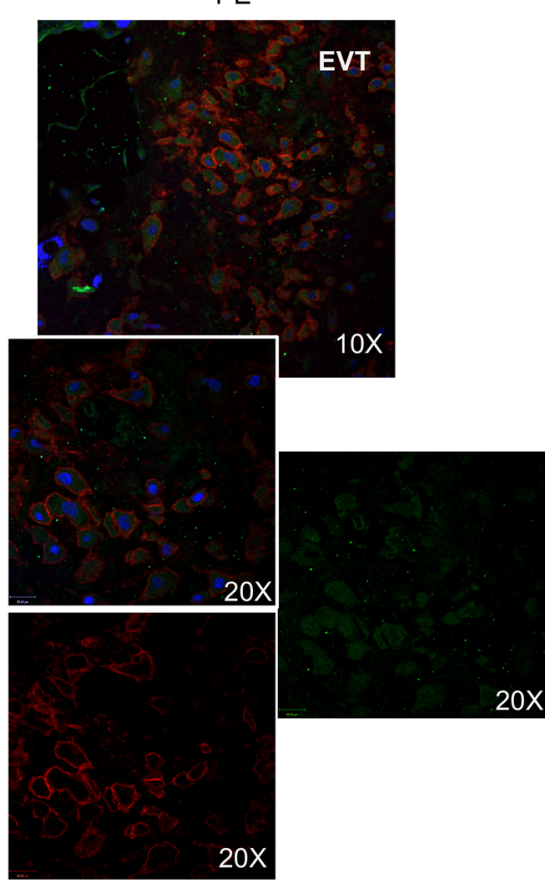

AMOT HLA-G DAPI

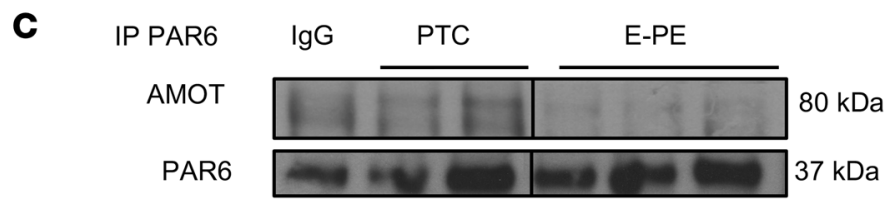

D

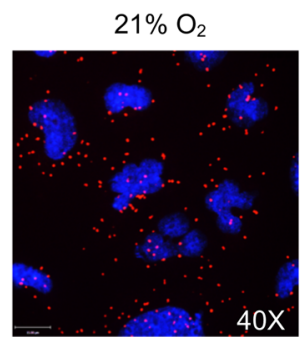

$3 \% \mathrm{O}_{2}$

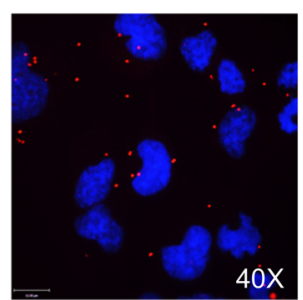

Negative Control minus probe

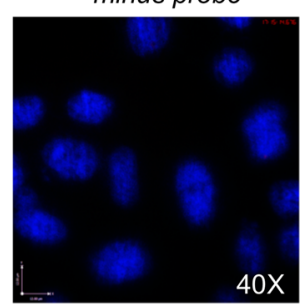

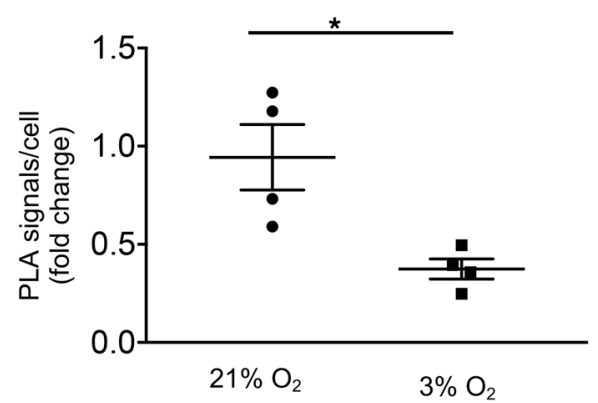

Figure 4. AMOT expression and localization are impaired in preeclampsia. (A) Representative Western blot (WB) of AMOT and associated densitometry in placental tissue lysates from normotensive preterm control (PTC) and early-onset preeclamptic (E-PE) placentae. AMOT protein levels were normalized by Ponceau staining and expressed as fold change relative to PTC. ${ }^{* *} P<0.001$ by nonparametric Mann-Whitney $U$ test (PTC, $n=8 ; \mathrm{E}-\mathrm{PE}, n=12$ ). (B) Representative immunofluorescence images of AMOT (green) and HLA-C (red) in term control (TC), PTC, and E-PE placental tissue sections at the fetomaternal interface. EVT, extravillous trophoblast. Original magnification, $\times 10$ and $\times 20$ (inset). Anti-goat lgG was used as control (TC, $n=3 ; P T C, n=3 ; P E, n=3$ ). (C) IP of PAR6 followed by WB of AMOT in PTC and E-PE placentae (PTC, $n=6$; E-PE $n=5$ ). Lanes were run on the same gel but were noncontiguous. (D) Representative images depicting in situ proximity ligation assay (PLA) of AMOT and PAR6 interaction in HTR-8/SVneo cells cultured in 21\% and $3 \%$ oxygen. Negative control was PLA reaction missing the minus (-) PLA probe and nuclei were stained with DAPI (blue). Original magnification, $\times 40$. Data were quantified as number of PLA signals per nucleus (cell) and expressed as fold change relative to $21 \%$ control. ${ }^{*} P<0.05$ by nonparametric Mann-Whitney $U$ test $(n=4)$. 
A

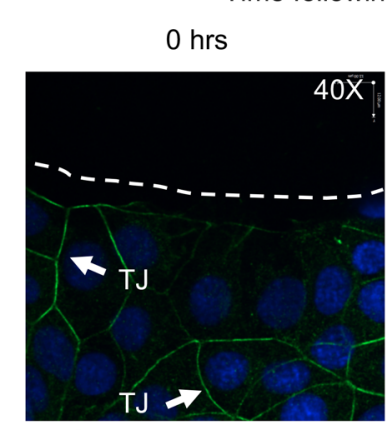

AMOT DAPI
B

AMOT 80 YFP

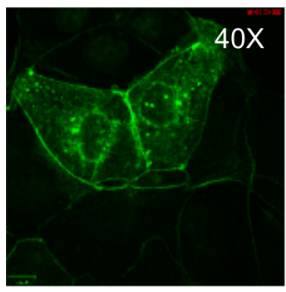

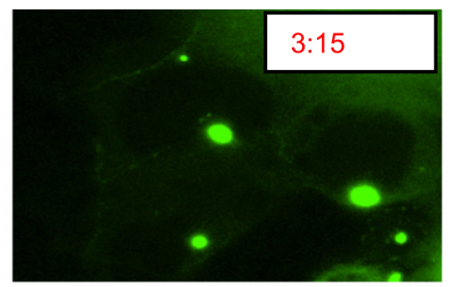
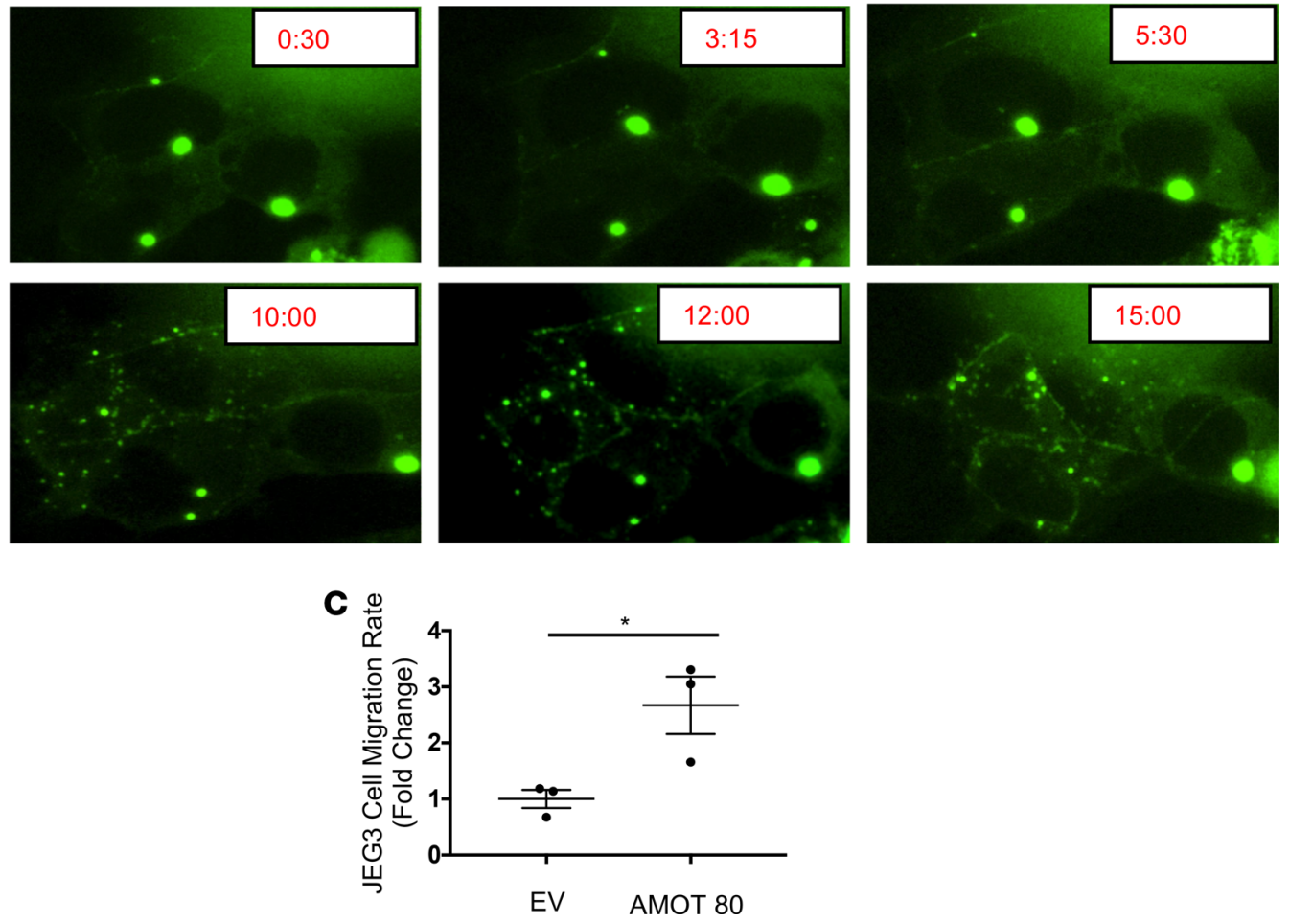

Figure 5. AMOT 80 promotes trophoblast cell migration. (A) Representative immunofluorescence images of AMOT (green) at 0 and 3 hours following wound initiation in confluent monolayers of JEG3 cells. Original magnification, $\times 40$. Nuclei were stained with DAPI (blue). Arrows indicate AMOT presence at the tight junction (TJ) or leading edge (LE), and asterisks indicate AMOT presence in cytoplasm. (B) Representative still pictures depicting localization of AMOT during cell migration in JEG3 cells overexpressing AMOT 80-YFP construct at various indicated time points. (C) Linear migration rate for JEC3 cells overexpressing AMOT 80-YFP expressed as fold change relative to empty vector (EV) control. ${ }^{*} P<0.05$ by 2 -tailed unpaired Student's $t$ test $(n=3)$.

between the intracellular tight junction and the cytoplasm, as elucidated by the live video (Supplemental Figure 3 and Supplemental Video 1) and various time-point snapshots (Figure 5B). Specifically, in the cytoplasm, AMOT is expressed as multiple, small punctate structures that are seen to dynamically redistribute throughout the cell during migration and division. Furthermore, the cells overexpressing AMOT accelerate their movement toward the wound, as quantification of migration rates revealed that JEG3 cells overexpressing AMOT 80-YFP migrated over 2-fold faster than empty vector controls (Figure 5C).

$A M O T$ is positively regulated by oxygen-dependent JMJD6. We have demonstrated a role for the JMJD6 lysyl hydroxylase, a novel oxygen sensor during human placenta development and in PE $(18,19)$. Silencing of JMJD6 in JEG3 cells resulted in decreased AMOT 130 and 80 protein levels compared with scrambled siRNA controls (Figure 6A). Correspondingly, overexpression of JMJD6 resulted in an increase in AMOT 130 and 80 protein levels compared with empty vector controls (Figure 6B), establishing JMJD6 as a positive regulator of AMOT. Considering that JMJD6 requires oxygen to elicit 

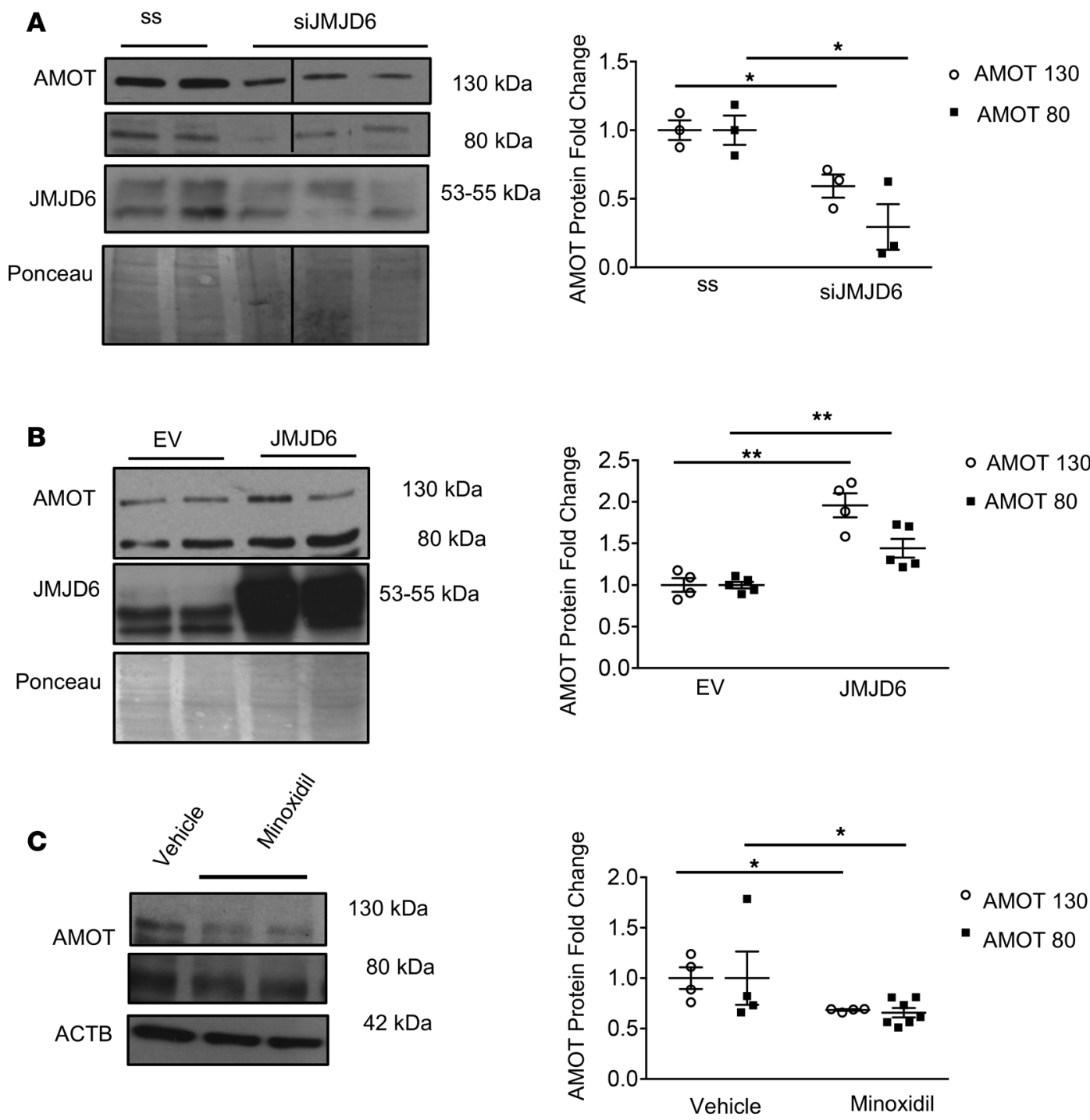

Figure 6. AMOT expression is positively regulated by oxygen-dependent JMJD6. (A) Representative Western blot (WB) of AMOT in JEG3 cells following silencing of JMJD6 (lanes were run on same gel but were noncontiguous). WB of JMJD6 in identical samples was run in parallel, contemporaneously along with AMOT blot to demonstrate JMJD6 silencing. Densitometric analysis of AMOT protein levels, which were normalized by Ponceau staining and expressed as fold change relative to scrambled siRNA (ss). ${ }^{*} P<0.05$ by 2 -tailed unpaired Student's $t$ test $(n=3)$. (B) Representative WB of JMJD6 and AMOT in JEG3 cells following overexpression of JMJD6. Densitometric analysis of AMOT protein levels, which were normalized by Ponceau staining and expressed as fold change relative to empty vector $(E V)$ control $(n=4)$. (C) Representative WB of AMOT in JEG3 cells following treatment $20 \mu M$ minoxidil for 48 hours. Densitometric analysis of AMOT protein levels, which were normalized to $\beta$-actin (ACTB) and expressed as fold change relative to vehicle control $(n=4) .{ }^{*} P<0.05$; ${ }^{* *} P<0.01$ by nonparametric Mann-Whitney $U$ test (B and $\left.\mathbf{C}\right)$.

its enzymatic functions, loss- and gain-of-function studies were performed at $21 \% \mathrm{O}_{2}$. To assess the contribution of JMJD6 to AMOT subcellular distribution and its role in cell migration, IF analysis of AMOT in HTR-8/SVneo cells was performed. Importantly, the stimulatory effect of AMOT 80 seen in JEG3 cell migration was confirmed in HTR-8/SVneo cells (Supplemental Figure 4, A and B, and Supplemental Videos 2 and 3). Silencing of JMJD6 in HTR-8/SVneo cells provoked a marked reduction of AMOT fibers in the cytoplasm and at the tight junction (Figure 7A). Conversely, overexpression of WT JMJD6 in HTR-8/SVneo cells promoted AMOT localization to the cytoplasm in fiber-like structures (Figure 7B) where it colocalized with F-actin (Figure 7B). In contrast, overexpression of a mutant JMJD6 construct with an impaired JMJC catalytic domain abrogated cytoplasmic AMOT fibers, and its colocalization with F-actin altogether (Figure 7B), suggesting this catalytic domain on JMJD6 may be important for its regulatory control over AMOT. The effect of JMJD6 overexpression on cell migration was next investigated. Following wound healing assay, HTR-8/SVneo cells overexpressing JMJD6 
A

ssRNA
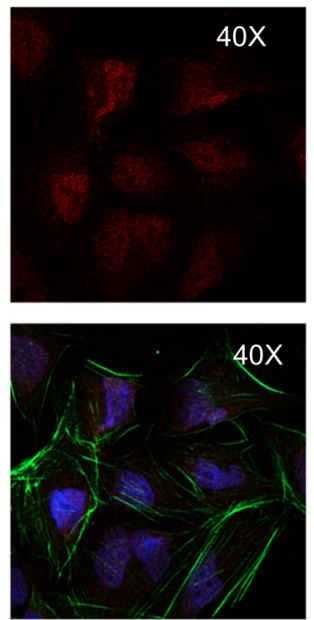

JMJD6
siJMJD6
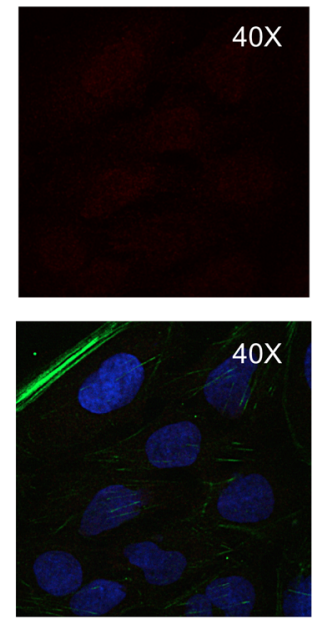

AMOT DAPI
B
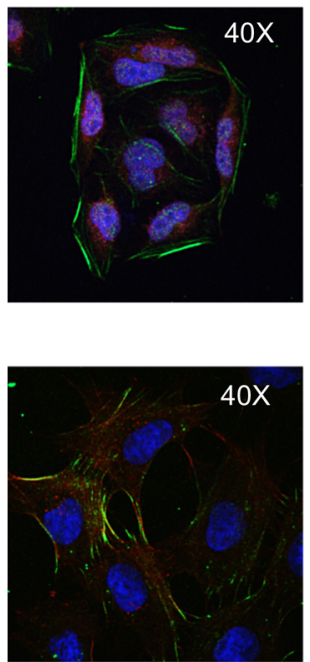

$\mathrm{JMJD6}_{\mathrm{WT}}$

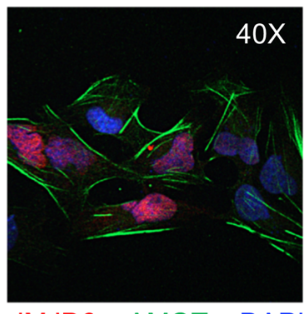

JMJD6 AMOT DAPI

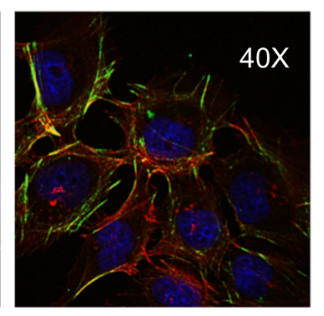

Phalloidin AMOT DAPI
$\mathrm{JMJD6}_{\text {mut }}$
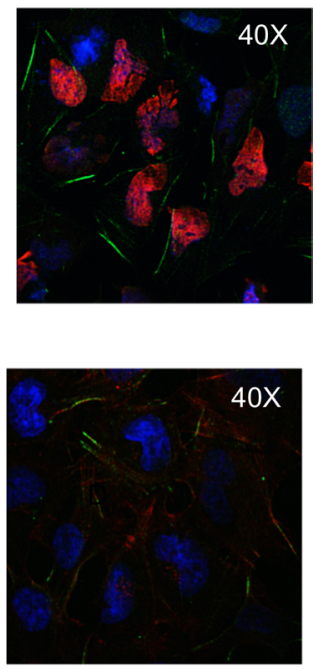

$40 X$
C

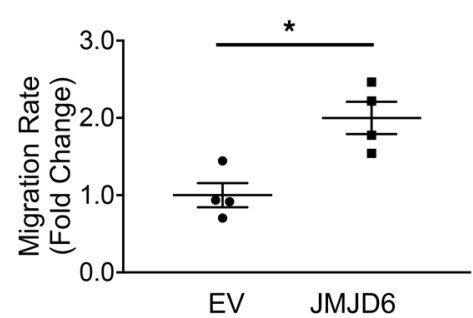

D

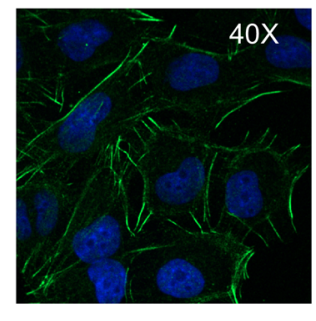

Minoxidil

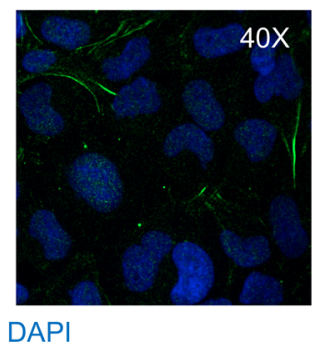

E

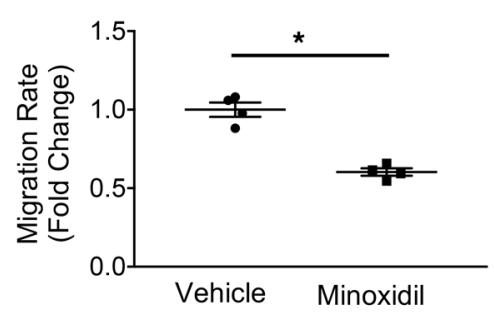

Figure 7. JMJD6 regulates AMOT subcellular localization and its lysyl hydroxylation in migratory HTR-8/SVneo cells. (A) Representative immunofluorescence (IF) images of AMOT (green) and JMJD6 (red) in HTR-8/SVneo cells following silencing of JMJD6 ( $n=3$ independent experiments). (B) Representative IF images of (i) AMOT and JMJD6, and (ii) AMOT and phalloidin in HTR-8/SVneo cells following overexpression of JMJD6 and JMJD6 with mutated catalytic JMJC domain $(n=4)$. (C) Linear migration rate for HTR-8/SVneo cells overexpressing JMJD6 expressed as fold change relative to empty vector (EV) control ( $n$ =4). (D) Representative IF images of AMOT in HTR-8/SVneo cells following treatment with $20 \mu \mathrm{M}$ minoxidil for 48 hours $(n=4)$. (E) Linear migration rate for HTR-8/SVneo cells treated with $20 \mu \mathrm{M}$ minoxidil for 48 hours expressed as fold change relative to vehicle control $(n=4) .{ }^{*} P<0.05$ by nonparametric Mann-Whitney $U$ test. Original magnification, $\times 40$ (all images).

migrated 1.5-fold faster than empty vector controls, underscoring a direct effect of JMJD6 on trophoblast cell migration (Figure 7C, Supplemental Figure 4C, and Supplemental Videos 4 and 5).

AMOT is subject to JMJD6-mediated lysyl hydroxylation. At its catalytic domain, JMJD6 elicits dual enzymatic function as both an arginine demethylase and a lysyl hydroxylase $(37,38)$. To assess the potential consequence of lysyl hydroxylation on AMOT, we utilized minoxidil, a widely employed pharmacological inhibitor of lysyl hydroxylation. Treatment of JEG3 cells with $20 \mu \mathrm{M}$ minoxidil led to a decrease of both AMOT 130 and 80 protein levels, compared with vehicle controls (Figure 6C). IF analysis showed that treatment of HTR-8/ SVneo cells with $20 \mu \mathrm{M}$ minoxidil reduced AMOT fluorescence and localization of cytoplasmic fibers in comparison with vehicle controls (Figure 7D). Further, minoxidil treatment significantly reduced the migration rate of HTR-8/SVneo cells during wound healing assay (Figure 7E). Collectively, these data suggest that lysyl hydroxylation as a posttranslational modification is important for AMOT content, its subcellular redistribution, and trophoblast migration. Using the RF-hydroxysite in silico analysis tool that predicts probability of hydroxylation at various lysine residues (39), we assessed both AMOT 130 and AMOT 80 protein sequences for potential lysyl hydroxylation. Analysis revealed a shared lysine residue found on AMOT 130 (position 758) and AMOT 80 (position 349) with a score of $100 \%$ probability of lysyl hydroxylation (Figure 8). Several other lysine residues were provided in the probability analysis report; however, none of these other lysine residues scored $100 \%$ probability of hydroxylation. A short AMOT peptide encompassing this $100 \%$ probability lysine residue was generated to assess whether JMJD6 directly hydroxylates AMOT at this lysine residue. 
$\mathrm{AMOT}_{753-762}$ peptide
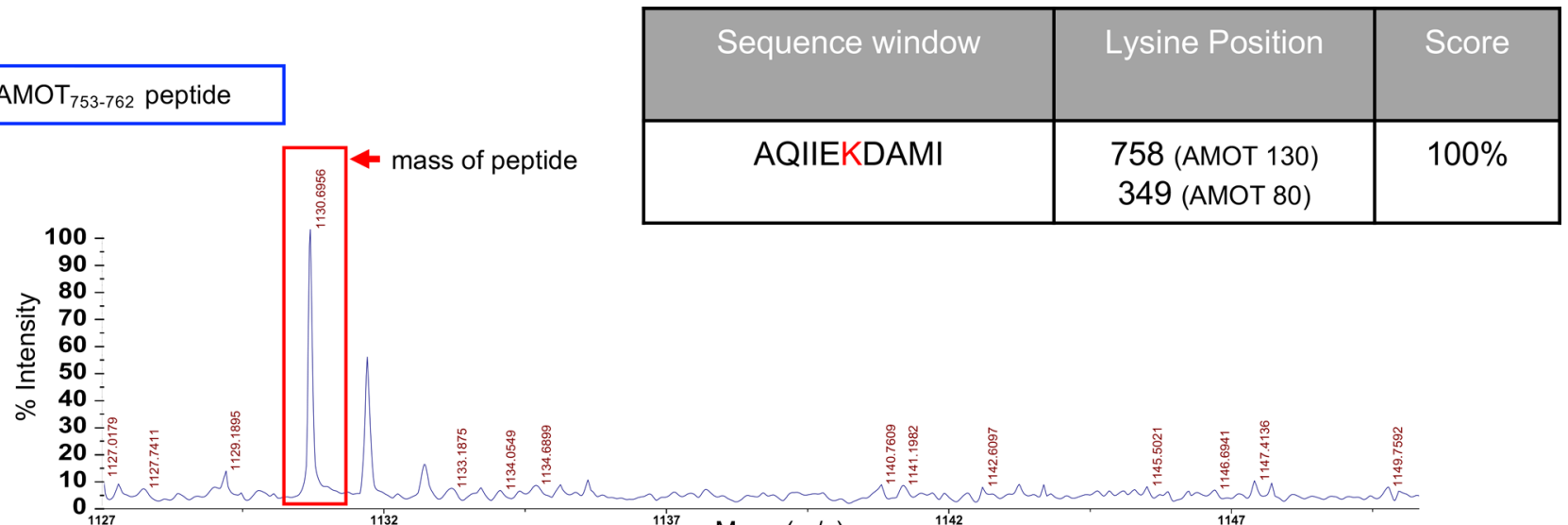

$\mathbf{O}_{1127}$

AMOT $_{753-762}$ peptide + JMJD6 enzyme

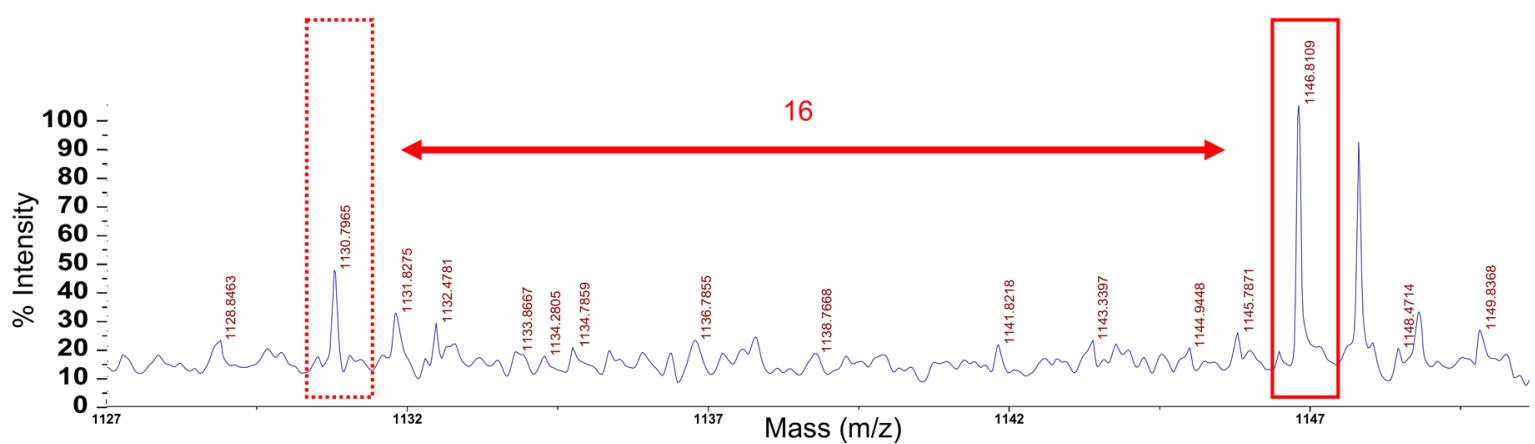

Figure 8. JMJD6 mediates lysyl hydroxylation of AMOT. Results from RF-Hydroxysite in silico analysis showing a peptide sequence window from AMOT 130 and AMOT 80 with a lysine residue (K) that scored $100 \%$ probability of lysyl hydroxylation: position 758 on AMOT 130 and position 349 on AMOT 80. Mass spectrometric profiles of AMOT peptide following in vitro reaction in the absence (top panel) and presence (bottom panel) of JMJD6 enzyme ( $n=3$ ).

Following an in vitro enzyme reaction with JMJD6 and necessary cofactors, the peptide mass profile was investigated by MALDI-TOF mass spectrometry. In the absence of JMJD6 enzyme, the primary peak is at 1,130 mass units, corresponding to the mass of the peptide (Figure 8, upper panel). However, after incubation with JMJD6 enzyme, the primary peak appears at 1,146 mass units. This shift in mass corresponds to the molecular mass of a hydroxyl group (16 mass units) (Figure 8, lower panel). This finding demonstrates that JMJD6 executes lysyl hydroxylation at this residue found within AMOT's peptide sequence, suggesting a direct posttranslational regulation of AMOT by JMJD6.

\section{Discussion}

The present study is the first to our knowledge to demonstrate an oxygen-dependent regulation of AMOT. We show that lysyl hydroxylation of AMOT via oxygen-dependent JMJD6 is an important posttranslational modification required for AMOT expression, localization, and importantly, its function in trophoblast cell migration - a cellular event that goes awry in PE. Further, we discovered what we believe is a novel association between polarity proteins AMOT and PAR6, and showed the AMOT/PAR6 interaction and AMOT localization to be regulated by both SMAD-dependent and SMAD-independent TGF- $\beta$ signaling. Of clinical relevance, our findings of reduced AMOT expression and association of AMOT with PAR6 in E-PE suggest that deficiency of this scaffolding protein may contribute to the shallow trophoblast migration/invasion that typifies this disease.

A role for AMOT in trophoblast cell migration during human placenta development. During early placenta development, proliferative CTs differentiate into migratory and invasive EVTs that populate the intermediate and distal regions of anchoring villi. This differentiation process is key to the establishment of the uteroplacental circulation and a successful pregnancy. A recent study demonstrated the earlier transition 
of CT to proximal column EVT to be propagated by low oxygen tension via HIF-1 $\alpha$ (40). Yet, studies in explants and isolated trophoblast cells from the first trimester have consistently demonstrated that low oxygen inhibits invasion $(10,29,41)$. As such, it appears that EVT differentiation may occur in 2 distinct processes regulated by oxygen, where during early implantation low oxygen initiates differentiation of CT to the EVT lineage, while a higher oxygen gradient is required to promote maturation of proximal EVTs to invasive distal EVTs. Our findings support the role of low oxygen in the initial CT to EVT transition because AMOT, whose expression is downregulated by hypoxia, is in fact absent in the proximal EVT column. Additionally, we demonstrate temporal and spatial changes in AMOT expression that coincide with increases in oxygen tension, and importantly provide evidence of a selective expression and localization of AMOT to the migratory EVTs comprising the anchoring column, specifically at their cell boundaries. AMOT's involvement in placenta development is further highlighted by mouse Amot-KO studies that display embryonic lethality at E11-11.5, a gestational point critical for completion of placenta development in mice (6). Our findings of AMOT 80 regulation of trophoblast cell migration are in line with studies in endothelial and MDCK epithelial cell lines, which showed that AMOT 80, but not AMOT 130, overexpression significantly increased migration rates $(25,42)$. Furthermore, our data showing AMOT redistribution from tight junctions to the cytoplasm in migrating cells, in conjunction with those demonstrating that AMOT 80 overexpression decreases levels of RhoA (a stabilizer of intracellular tight junctions and polarity; see ref. 36), altogether support a role for AMOT in regulating the tight-junction stability of trophoblast cells amid maintenance of cell polarity.

In addition to cell polarity regulation, AMOT has a well-characterized role in Hippo pathway regulation whereby its direct interaction with transcriptional coactivators YAP and TAZ control downstream cellular processes such as cell proliferation and differentiation. In fact, it is AMOT's association with TAZ that has been implicated in lineage differentiation in the early embryo $(8,43)$. However, the role of the AMOT/TAZ axis in trophoblast cell migration in the first-trimester human placenta remains unknown. Future studies are warranted to examine AMOT and TAZ interaction in EVT migration and/or invasion.

Delineating the complex regulation of AMOT by TGF- $\beta$ signaling pathways. TGF- $\beta$ is an integral regulator of epithelial to mesenchymal transition (EMT) processes, as its addition to epithelial cells in culture is accepted as a convenient way to induce EMT in epithelial cells from various cancers (44). Importantly, TGF- $\beta$ has been reported to regulate trophoblast cell migration during early human placenta development $(10,28,45)$. To our knowledge, the present study is the first to show TGF- $\beta$ as a regulator of AMOT expression and cytoplasmic redistribution. Interestingly, AMOT isoforms have been shown to contribute to cell migration via different modes of action. Although AMOT 80 regulates tight junction integrity via interactions with GTPase-activating protein Rich1 and with components of Crumbs polarity complex (2), AMOT 130 preferentially binds to F-actin, thereby regulating YAP- and TAZ-mediated transcription of genes involved in proliferation and EMT $(46,47)$. Furthermore, AMOT 130 and F-actin interactions also facilitate changes in cell shape (24), integrity of tight junctions, and actin remodeling (48), all of which promote cell motility events (49). Our data in JEG3 and HTR-8/SVneo trophoblast cells corroborate the latter findings, as we show that TGF- $\beta$ promotes AMOT binding to the F-actin cytoskeleton, leading to disruption of tight junctions and changes in cell shape, altogether contributing to cell migration. These findings were confirmed in first-trimester explants where treatment with AS-TGF- $\beta 3$ maintained AMOT localization at the boundaries of HLA-G-positive EVTs. Previous work from our lab has established that inhibition of TGF- $\beta 3$ in villous explants induced formation of migratory EVT columns (29). This observation, in conjunction with our current findings of AMOT localization to HLA-G ${ }^{+}$EVTs upon TGF- $\beta 3$ inhibition, altogether point to a TGF- $\beta$-dependent role of AMOT during EVT migration. With the recent advent of protocols for isolation and maintenance of primary EVTs (50), as well as availability of newly established trophoblast stem cell lines (51), future studies are encouraged to confirm our findings of AMOT's role in cell migration in these primary cell models.

Studies have revealed a novel lipid-binding domain on AMOT that targets it along with associated apical polarity proteins to the endosomal recycling compartment $(2,52)$. Considering the reduction in AMOT protein levels following TGF- $\beta$ exposure, it is plausible that TGF- $\beta$-induced AMOT cytoplasmic localization may result in the degradation of this protein, further contributing to alterations in cell polarity. The current study demonstrates that TGF- $\beta 1 / 3$-mediated redistribution of AMOT in JEG3 cells is reliant on Smad-dependent signaling. Previous studies have reported JEG3 cells to be resistant to the effects of TGF- $\beta$ as a mechanism to escape normal regulatory control of cell invasion due to poor 
Smad3 expression $(53,54)$. However, a recent study has shown that JEG3 cells are responsive to TGF- $\beta$ exposure through Smad2 signaling (28), which is confirmed by the present investigation.

Implications of an AMOT 80 and PAR6 interaction. Maintenance and loss of apical-basolateral polarity is reliant on dynamic interactions between polarity complex proteins including Par/aPKC (33), and these interactions are facilitated by specific interaction motifs. For instance, the $\mathrm{C}$-terminal PDZ-binding domain on AMOT is critical for its interaction with the Pals1/Patj polarity complex and its localization at the tight junction $(2,4)$. Considering PAR6 presence at tight junctions is required for their maintenance, discovery of an AMOT-PAR6 association within the cytoplasm following TGF- $\beta 1 / 3$ treatment indicates that AMOT effectively sequesters PAR6 protein away from the tight junctions, thereby contributing to their dissolution and loss of apical-basolateral cell polarity. In the developing placenta, TGF- $\beta$ acts in a complex manner because it stimulates various cellular functions in different trophoblast subpopulations (28). This can be attributed to diverse TGF- $\beta$ signaling pathways being activated in a cell-specific manner throughout development. Having established a role for AMOT in (a) Smad-dependent and (b) PAR6-mediated TGF- $\beta$ signaling pathways further underscores the complexity of TGF- $\beta$ signaling in the human placenta. The selective interaction of PAR6 with AMOT 80 is not surprising, as in other studies AMOT 80 has been reported to be the predominant AMOT isoform involved in interactions with other scaffolding polarity proteins (2). Analysis of the binding domains required for this interaction revealed the PDZ domain on AMOT 80 to be important for PAR6 binding to AMOT 80, but not AMOT 130, in line with studies that show mutations in the C-terminal PDZ domain of AMOT 80 impairs its ability to localize to tight junction proteins such as MUPP1 (4) and the Pals1/Patj/Par3 complex (2). Further, the PDZ-binding domain on AMOT 80 has proven to be essential for its function in cell migration, as cells expressing a PDZ-mutant form of AMOT exhibited defective migration (55). Considering that the present study and others have emphasized the role of AMOT 80 in cell migration versus the role of AMOT 130 in cell shape changes $(24,25)$, it is possible that the AMOT 80-PAR6 interaction is primarily involved in promotion of cell migration via cell polarity changes.

JMJD6 lysyl hydroxylase positively regulates AMOT. Incumbent on oxygen availability, JMJD6 has been shown to affect diverse cellular processes, including cell migration, by promoting alterations in gene transcription and/or protein stability via its two main enzymatic functions: lysine hydroxylation and histone demethylation $(19,23,37,38)$. In this study, we demonstrate a role for JMJD6 in trophoblast cell migration and provide evidence suggesting this function to be dependent on JMJD6 lysyl hydroxylase activity. We also establish JMJD6 lysyl hydroxylase as a new regulator of AMOT levels and localization in migratory cells. Hence, we postulate that the JMJD6-directed promotion of cell migration is occurring via AMOT. Posttranslational modifications of AMOT have only recently begun to be uncovered, where omics-type approaches identified modifications of AMOT to include phosphorylation, ubiquitination, acetylation, and glycosylation (56). The ability of posttranslational modifications to impact AMOT localization and function is demonstrated by studies showing how LATS1/2 kinase-dependent phosphorylation of AMOT 130 at serine 175 inhibits its binding to the F-actin cytoskeleton, impairs cell migration and angiogenesis, while freeing it to interact with YAP and TAZ $(8,57)$. The present study adds to the current knowledge by identifying AMOT as a target for JMJD6-mediated lysyl hydroxylation. Additionally, we show JMJD6-mediated lysyl hydroxylation to be important for AMOT stability and subcellular redistribution, as impediments in lysyl hydroxylase activity or overexpressing JMJD6 with a mutated catalytic domain reduced AMOT protein levels and altered deposition of AMOT fibers in the cytoplasm.

Implications of decreased levels of AMOT in PE. Aside from investigations implicating a role for AMOT in various cancers $(58,59)$, the contribution of AMOT to the pathogenesis and progression of human disease is poorly understood. As such, this study is the first to our knowledge to address AMOT deficiency in PE, a human pregnancy-associated disorder characterized by insufficient EVT invasion of spiral arteries and impaired trophoblast turnover. It is likely that the drastic reduction of total AMOT due to reduced oxygenation in $\mathrm{PE}$ impacts the ability of EVTs to acquire the migratory and invasive functionality required to invade the maternal spiral arteries. Gene expression analysis studies have demonstrated that first-trimester differentiation of anchorage-dependent CTs into motile EVTs is analogous to an EMT (60), and this process becomes constrained in the third trimester to allow EVTs to reach a metastable state (61). Yet, in the pathological scenario of abnormally invasive placentae, third-trimester EVTs propagate their first-trimester-like phenotype and retain their mesenchymal properties that provokes the hyperinvasive phenotype (61). Our data demonstrating a deficiency of AMOT, which we show to be promigratory for EVTs, in PE supports the notion that in the hypoinvasive setting, EVT maturation into a migratory 
phenotype may be attenuated, and ultimately nonfunctional EVTs recourse to a noninvasive phenotype. Additionally, disruption of the interaction described in this study between AMOT 80 and PAR6 in PE may also exacerbate the immature trophoblast phenotype, as we propose that the collective AMOT and PAR6 relocalization to the cytoplasm may be disassociating vital polarity complexes, thereby contributing to cell migration. Work from our lab has established PAR6 as a negative regulator of CT fusion by maintaining intracellular tight junctions, and further found that its elevated expression in PE may contribute to the aberrant trophoblast cell turnover typical of this pathology (32).

Changes in oxygen tension and TGF- $\beta$ signaling are intricately linked during placenta development, and persistence of low oxygen and elevated TGF- $\beta$ signaling are molecular characteristics of PE $(10,28$, 29). The present data demonstrating reduced AMOT protein levels following treatment with TGF- $\beta 1 / 3$, exposure to $3 \% \mathrm{O}_{2}$, and during early placentation (5-9 weeks of gestation), when oxygen tension is low, are consistent with the reduction of AMOT seen in PE. This suggests that the impaired AMOT levels and localization in PE could be a result of prolonged low oxygen exposure and increased downstream TGF- $\beta$ signaling. No studies to our knowledge have investigated the impact of oxygen on AMOT expression; however, short exposure to low oxygen in early human placental villi showed upregulation of several genes involved in angiogenesis, one of them being AMOTL2 (62). Likewise, exposure of HeLa cells to low oxygen increased a spliced form of AMOTL2 (63). However, these studies focused solely on AMOTL2. Thus, it is plausible that changes in oxygen may differentially affect the expression of diverse motin proteins in a cell-dependent manner. Furthermore, JMJD6 enzymatic function is impaired in PE because of lowered oxygen and intracellular iron availability $(18,19)$. Thus, impaired JMJD6 regulation of AMOT in PE could account for disrupted AMOT protein levels and altered distribution in EVTs.

Tankyrase inhibitors and their effect on AMOT protein stabilization is currently exploited in cancer investigations, as increasing AMOT stability is shown to inhibit downstream Hippo pathway signaling via YAP, thereby suppressing YAP-mediated oncogenesis $(30,64)$. We introduce utility for tankyrase inhibitors in trophoblast cells by demonstrating that inhibition of tankyrase activity halts the oxygen-mediated downregulation of AMOT. These findings suggest tankyrase inhibition as a potential rescue for AMOT deficiency in PE, which is characterized by low oxygen. Furthermore, it rationalizes further investigation into the role of AMOT and Hippo pathway effectors in trophoblast migration.

The findings of this study are summarized in the graphical abstract. During normal placentation, TGF- $\beta$-mediated redistribution of AMOT to the F-actin scaffold, its interaction with PAR6, and JMJD6-mediated regulation of AMOT expression and localization altogether favor AMOT's role in promoting trophoblast cell migration. In PE, however, the chronic low oxygen environment upregulates levels of TGF- $\beta 3$ that subsequently downregulate AMOT levels and disrupt the AMOT and PAR6 interaction. Most notably, the lack of molecular oxygen in PE renders JMJD6 inactive and unable to regulate AMOT expression and function. This ultimately contributes to the downregulation of AMOT, leading to the impaired trophoblast migration phenotype seen in $\mathrm{PE}$.

\section{Methods}

Tissue collection. Placentae from early gestation (5-16 weeks, $n=49)$ were obtained following elective termination of pregnancy. Preterm $(n=15)$, term $(n=3)$, and E-PE $(n=19)$ placentae were obtained through The Research Centre for Women's and Infant's Health (RCWIH) BioBank at Mount Sinai Hospital, Toronto. PE placentae were selected according to American College of Obstetricians and Gynecologists (ACOG) guidelines, and definition of E-PE included delivery prior to 34 weeks of gestation. Patients with chronic hypertension, diabetes, infection, and kidney disease were excluded. Normotensive, age-matched placentae from healthy preterm (PTC) deliveries exhibiting no signs of placental disease or infection were used as controls. Typical cause for PTC deliveries included cervical incompetence, idiopathic labor, and PTC premature rupture of membranes. Placenta samples were either snap frozen for RNA and protein analysis or fixed in paraformaldehyde (PFA) for subsequent histological and immunohistochemical or IF imaging and analyses. Clinical features of PE and E-PE are summarized in Table 1.

Cell culture and treatments. JEG3 human choriocarcinoma cells (ATCC, HTB-36) were cultured in Eagle's minimal essential medium (EMEM), and HTR-8/SVneo human trophoblast cells (ATTC, CRL3271) were cultured in RPMI, supplemented with $10 \% \mathrm{FBS}$ and $1 \%$ penicillin-streptomycin (10,000 units/ $\mathrm{ml})$ and maintained at $37^{\circ} \mathrm{C}$ under standard culture conditions $\left(5 \% \mathrm{CO}_{2}, 21 \% \mathrm{O}_{2}\right)$. For 6-well plates, JEG3 cells were seeded overnight at $2.0 \times 10^{5}$ cells/well to a confluence of $70 \%$ before treatments. For Nunc 
Table 1. Clinical features of PE and E-PE

\begin{tabular}{|c|c|c|c|c|}
\hline \multirow{2}{*}{ Clinical Parameter } & PTC & E-PE & $P$ value & TC \\
\hline & $n=15$ & $n=19$ & & $n=3$ \\
\hline Gestational Age at Delivery (weeks) & $30.2 \pm 0.9$ & $29.0 \pm 0.6$ & NS & $38.4 \pm 0.4$ \\
\hline \multirow{2}{*}{ Fetal Sex } & $42.85 \%$ & $40 \% \mathrm{~F}$ & NS & $60 \% \mathrm{~F}$ \\
\hline & F $57.14 \%$ M & $60 \% \mathrm{M}$ & & $30 \% \mathrm{M}$ \\
\hline Blood Pressure (mmHg, S/D) & S: $107.2 \pm 3.6$ & $S: 159.6 \pm 5.5^{B}$ & $\mathrm{~S}: P<0.001$ & S: $116.6 \pm 3.3$ \\
\hline \multirow{2}{*}{ Mode of Delivery (\%) } & $62.5 \%$ VD & $25 \%$ VD & NS & $30 \%$ SVD \\
\hline & $37.5 \%$ CS & $75 \%$ CS & & $60 \%$ CS \\
\hline
\end{tabular}

Data are presented as mean \pm SEM. ${ }^{A} P<0.05 .{ }^{B} P<0.001$. A nonparametric Mann-Whitney test was used when comparing gestational age at delivery, fetal birth weight, and blood pressure in E-PE to PTC, and a $\chi^{2}$ test was used when comparing fetal sex and mode of delivery in E-PE and PTC. Reference column of term controls (TC) for all parameters is on the right. NS, nonsignificant; PTC, preterm controls; E-PE, early-onset preeclampsia; F, female; M, male; S, systolic; D, diastolic; CS, caesarian section; VD, vaginal delivery.

Lab-Tek II 0.7- $\mathrm{cm}^{2} 8$-well chamber slides (Thermo Fisher Scientific), cells were seeded overnight at $1.5 \times$ $10^{4}$ cells/well before treatments. For 1.0- $\mathrm{cm}^{2} 8$-well u-slides (ibidi Inc), cells were seeded overnight at 9.0 $\times 10^{4}$ cells/well before treatments and live-cell imaging. JEG3 cells were treated for 24 hours with $10 \mathrm{ng} /$ $\mathrm{ml}$ TGF- $\beta 1 / \mathrm{B} 3$ (R\&D Systems) or vehicle control $(4 \mathrm{mM} \mathrm{HCl}$ containing $1 \mathrm{mg} / \mathrm{ml}$ bovine serum albumin [BSA]); or $5 \mu \mathrm{M}$ SB-431542 (Sigma-Aldrich) or vehicle control (DMSO). For SB-431542 plus TGF- $\beta$ treatments, SB-431542 inhibitor was added 30 minutes prior to TGF- $\beta$ treatment. For lysyl hydroxylation inhibition, JEG3 or HTR-8/SVneo cells were treated for 48 hours with $20 \mu \mathrm{M}$ minoxidil (Sigma-Aldrich), or $40 \mu \mathrm{l}$ vehicle control (95\% vol/vol ethanol). For low oxygen treatment, JEG3 cells or HTR-8/SVneo cells were grown in $21 \% \mathrm{O}_{2}$ normoxic conditions prior to incubation in $3 \% \mathrm{O}_{2}\left(5 \% \mathrm{CO}_{2}, 92 \% \mathrm{~N}_{2}\right)$ for 24 hours. For tankyrase inhibition experiments, HTR-8/SVneo cells were treated for 24 hours with $10 \mu \mathrm{M}$ XAV939 (Sigma-Aldrich, X3004).

Human chorionic villous explant culture and antisense oligonucleotide treatment. Human villous explants were established from first-trimester human placental tissue (5-13 weeks of gestation) and treated with $10 \mu \mathrm{M}$ control sense or antisense oligonucleotides against HIF1A or TGF- $\beta 3$ as previously described $(10,29)$.

siRNA transfections. JEG3 or HTR-8/SVneo cells were grown to 50\%-60\% confluence in 6-well plates prior to 24-hour incubation with siRNA transfection mix containing $30 \mathrm{nM}$ Silencer Select siRNA targeted against JMJD6 or scrambled siRNA sequences (control), $200 \mu 1$ of jetPRIME buffer, and $4 \mu 1$ of jetPRIME (Polyplus transfection) as previously reported (18).

Plasmid DNA constructs and transfections. The following plasmids were obtained from Addgene: AMOT 130 (HA-AMOT p130, 32821); AMOT 130PDZ (HA-AMOT p130 delta EYLI, 32826); AMOT 130 delta coiled-coil (HA-AMOT p130 delta CC, 32827) (47); JMJD6 (p6352 MSCV-CMV-Flag-HA-JMJD6, 31358) (65); and PAR6 (pCMV5 T7-PAR6 WT T7 tagged, 24649) (35). pcDNA3 (c-Flag pcDNA3, 20011) was used as empty vector control for all AMOT plasmids (66). MSCV PIG (Puro IRES GFP, 18751) served as the empty vector control for JMJD6 plasmids. Mutated JMJD6 construct (mut JMJC) was generated using site-directed mutagenesis of histidine 187 to alanine (H187A) and aspartic acid to alanine (D189A). The following plasmids were obtained through OpenFreezer Laboratory Reagent Tracking and Workflow Management System, a reagent repository at Mount Sinai Hospital, Toronto: AMOT 80 (angiomotin/ Kiaa1071/FL, pLP Triple Flag SD, ID V988-Pawson Lab); AMOT 80-YFP (angiomotin FL, pLP EYFP C1, ID V1489-Clontech); and AMOT 80 delta PDZ (angiomotin/Kiaa1071/-YLI, pLP Triple Flag SD, ID V1145-Pawson Lab) (2). JEG3 or HTR-8/SVneo cells were grown to $60 \%-80 \%$ confluence in 6-well plates prior to 24 -hour incubation with $1 \mu \mathrm{g}$ of plasmid DNA, $200 \mu \mathrm{l}$ of jetPRIME, and $4 \mu \mathrm{l}$ of jetPRIME reagent. In 8-well chamber slides, $0.5 \mu \mathrm{g}$ of plasmid DNA was added to $25 \mu$ of jetPRIME buffer and $1 \mu 1$ of jetPRIME reagent. Media were replaced 6 hours following transfection.

Wound healing assay. JEG3 and HTR-8/SVneo cells plated on coverslips in 6-well plates, or 8-well ibidi u-slides, were grown until complete confluence and scratched with a sterile pipet tip spanning the length of 
the coverslip or slide. Cells were treated or transfected 24-48 hours prior to wound initiation to establish biological effect and confluence. Cells were gently rinsed with PBS to remove cell debris following scratch, and fresh $2 \mathrm{ml}$ of media was added to each well. In 6-well plates, cells were fixed for IF immediately following wound initiation ( 0 hours), as well as 3 and 24 hours after the scratch by addition of 3.7\% (vol/ vol) formaldehyde for 15 minutes at room temperature. In 8-well ibidi u-slides, cells were placed under the time-lapse microscope immediately following wound initiation and imaged for 24 hours.

Time-lapse live cell imaging. JEG3 or HTR-8/SVneo cells seeded on 8-well ibidi u-slides were placed in a temperature- and $\mathrm{CO}_{2}$-regulated incubation chamber $\left(37^{\circ} \mathrm{C}, 5 \% \mathrm{CO}_{2}, 21 \% \mathrm{O}_{2}\right)$ and mounted onto the microscope stage. Images were acquired with a $\times 10$ or $\times 20$ Leica objective, and captured every 15 minutes for 24 hours. Migration rates were determined by measuring the area of the scratch at various time points using Volocity Imaging software (Quorum Technologies) and plotting area versus time on a scatter plot in Microsoft Excel. A trendline was applied to the scatter plot, and the slope of the line was used as the migration rate.

WB analysis. WB and Bradford protein assay (Bio-Rad) were performed as previously described (67). Ponceau stain, $\beta$-actin, or $\alpha$-tubulin was used as a loading control. Densitometric analysis was performed using ImageJ software (NIH).

Antibodies. Primary antibodies used include AMOT (Santa Cruz Biotechnology, L-16, sc-82493, goat polyclonal [IF 1:1000]); AMOT (Thermo Fisher Scientific, PA5-31196, rabbit polyclonal [WB 1:1000]); AMOT (Cell Signaling Technology, 431305, D204H, rabbit polyclonal [IF/PLA 1:100]); $\alpha$-tubulin (Santa Cruz Biotechnology, P16, sc-31779, goat polyclonal [WB 1:500]); $\beta$-actin (Santa Cruz Biotechnology, C-4, sc-47778, mouse monoclonal [WB 1:2000]); HLA-G (Exbio 4H84, mouse monoclonal [IF 1:300]); JMJD6 (Abcam Inc, ab64575, goat polyclonal [WB 1:500]); PAR6A (Santa Cruz Biotechnology, C-3, sc-365323, mouse monoclonal [WB 1:500; IF/PLA 1:100]); PAR6A (Aviva Systems Biology, ARP42883_P050, rabbit polyclonal [WB 1:600]); p-SMAD2 (Abcam Inc, ab53100, rabbit polyclonal [WB 1:800]); RhoA (Abcam Inc, ab54835, mouse monoclonal [WB 1:500; IF 1:200]); SMAD2 (Cell Signaling Technology, 3122, 86F7, rabbit monoclonal [WB 1:1000]); TAZ (Santa Cruz Biotechnology, 1F1, sc-293183, mouse monoclonal [IF 1:300]); and ZO-1 (Santa Cruz Biotechnology, H-300, sc-10804, rabbit polyclonal [IF 1:200]). Phalloidin probe conjugated to photostable, red fluorescent Alexa Fluor 594 was used to stain the F-actin cytoskeleton. Secondary antibodies used in WB analyses were obtained from Santa Cruz Biotechnology and include horseradish peroxidase (HRP) conjugated to rabbit anti-mouse (1:2000), HRP goat anti-rabbit $(1: 1000)$, and HRP donkey anti-goat (1:1000). Secondary antibodies used in IF analyses were obtained from Thermo Fisher Scientific, used at a dilution of 1:300 and include: Alexa Fluor 488 donkey anti-rabbit (A21206), 594 donkey anti-rabbit (A-21207), 488 donkey anti-goat (A-11055), 594 donkey anti-goat (A-11058), 488 donkey anti-mouse (A-21202), and 594 donkey anti-mouse (A-21203).

$I P$. Procedures for IP were performed as previously described (67). Anti-PAR6 antibody ( $2 \mu \mathrm{g})$, or normal mouse IgG for control, was added to 200-300 $\mu \mathrm{g}$ of $1 \mu \mathrm{g} / \mu \mathrm{l}$ total cell or tissue lysate in RIPA buffer and incubated overnight at $4^{\circ} \mathrm{C}$. Immunocomplexes were centrifuged and successively washed with PBS and RIPA buffer prior to the final pellet being dissolved in $40 \mu 1$ of $2 \times$ SDS sample buffer. IP proteins were analyzed by WB.

$I F$. IF staining was performed as previously described (67). For tissue sections, $10 \mathrm{mM}$ sodium citrate ( $\mathrm{pH}$ 6.0) was used for antigen retrieval, $0.4 \%$ (vol/vol) Triton X-100 (Bioshop Canada Inc.) for tissue permeabilization, and $0.3 \%$ (vol/vol) Sudan Black in 70\% ethanol for 30 minutes to quench endogenous fluorescence. JEG3 and HTR-8/SVneo cells were permeabilized with $0.2 \%$ (vol/vol) Triton X-100. Sections were blocked at room temperature for 1 hour with 5\% (vol/vol) normal horse serum (NHS) (Sigma-Aldrich) prior to overnight incubation at $4^{\circ} \mathrm{C}$ with primary antibody diluted in equal parts blocking solution and antibody diluent $(0.4 \%$ [wt/vol] sodium azide, $0.625 \%$ [wt/vol] gelatin). Anti-mouse or -goat IgG was used as a negative control (Santa Cruz Biotechnology, sc-2025 or sc-2028). Sections were incubated with 4',6-diamino-2-phenylindole (DAPI, Sigma-Aldrich) and mounted using Immuno-Mount solution (Thermo Fisher Scientific). Images were captured using a Leica SD6000 spinning disk confocal microscope and quantified using Volocity Imaging software.

Immunohistochemistry. Immunohistochemical staining of placental tissue was performed as previously described (10). Antigen retrieval was performed the same way as in IF, and endogenous peroxidase activity was inhibited using 3\% (vol/vol) hydrogen peroxide in methanol for 30 minutes at room temperature. Blocking and primary antibody incubation was performed the same way as in IF. Biotinylated secondary antibody was added for 2 hours at room temperature, washed in PBS, and incubated in avidin-biotin-HRP 
complex from VectaStain Standard Kit (Vector Laboratories). Slides were then washed with PBS and incubated with DAB solution $\left(0.075 \%\right.$ [wt/vol] in $1 \times$ PBS solution containing $0.002 \%$ [vol/vol] $\left.\mathrm{H}_{2} \mathrm{O}_{2}\right)$. Slides were mounted using Surgipath micromount medium (Leica). Images were captured using an Olympus BX61 motorized light microscope system.

PLA. JEG3 and HTR-8/SVneo cells were cultured and treated on Nunc Lab-Tek II 8-well chamber slides (Thermo Fisher Scientific). Cells were fixed and permeabilized as described above, and all subsequent steps followed the Duolink PLA Fluorescence Protocol (Sigma-Aldrich). Cells were mounted with coverslips using in situ mounting medium with DAPI. Three negative controls were used: PLA reaction in the absence of either the AMOT or PAR6 antibody, in the absence of plus-PLA probe, and in the absence of both plus- and minus-PLA probes. Images were captured using a Leica SD6000 spinning disk confocal microscope and Volocity Imaging software. PLA quantification was performed manually by 2 independent assessors who were blinded to the treatment groups. Data are expressed as fold change of PLA signals normalized to number of nuclei.

LCM. LCM was performed on cryosections of first-trimester placentae (5-13 weeks of gestation) to isolate the villous ST/CT trophoblast layer, and the proximal and distal anchoring column EVTs as previously described (27).

$R N A$ isolation and real-time quantitative PCR. RNA was isolated from placental tissues using TRIzol reagent (Invitrogen) and $1 \mu \mathrm{g}$ was reverse transcribed into cDNA using qScript cDNA SuperMix following the manufacturer's protocol (Quanta Biosciences). PCR amplification of cDNA was conducted using the DNA Engine Opticon 2 System (MJ Research) following addition of $1 \mu \mathrm{l}$ of AMOT probe (Hs00611096_ m1, AMOT, TaqMan Assays-on-Demand from Applied Biosystems), $10 \mu 1$ of TaqMan Universal PCR Master Mix (Applied Biosystems), and $6 \mu$ of DEPC $\mathrm{H}_{2} \mathrm{O}$ to the converted cDNA (3 $\mu$ l). Ribosomal 18S RNA was used as a housekeeping gene and all AMOT C(T) values were normalized to $18 \mathrm{~S}$ using the comparative delta $\mathrm{C}(\mathrm{T})$ method.

In vitro JMJD6 hydroxylation reaction. Protein sequences for AMOT 130 (NCBI Ref Seq: NP_001106962.1) and AMOT 80 (NCBI Ref Seq: NP_573572.1) were input into in silico RF-Hydroxysite Prediction software in FASTA format (39), and the probability of lysine hydroxylation of AMOT was assessed. The following parameters were set in the search: Residue $=$ Lysine $(\mathrm{K})$; Window size $=15$; Score threshold $=0.6$. Using the protein sequence information for AMOT 130/80, a 10-aa peptide containing a 100\% probability lysine residue and flanking residues were identified and synthesized (CanPeptide Inc). The sequence of the synthesized AMOT peptide was AQIIEKDAMI, and its MW was determined to be 1,131.35 Da. For in vitro enzyme studies, 7.5 $\mu \mathrm{g}$ of AMOT peptide was incubated with 100 or $200 \mathrm{ng}$ of purified human recombinant JMJD6 enzyme (BPS Bioscience) in the presence of hydroxylation buffer (50 mM Tris pH 7.9, $50 \mathrm{mM} \mathrm{KCl}, 10 \mathrm{mM} \mathrm{MgCl}, 2 \mathrm{mM}$ ascorbate, $1 \mathrm{mM} \alpha$-ketoglutarate, $50 \mu \mathrm{M} \mathrm{Fe}^{2+}$ in $\mathrm{dH}_{2} \mathrm{O}$ ) at $37^{\circ} \mathrm{C}$ for 2 hours. Reactions were performed in a 24 -well plate, with a final volume of $100 \mu$. Reactions performed in the absence of JMJD6 enzyme served as a negative control. Following a 2-hour enzymatic reaction, reactions were collected and stored at $-20^{\circ} \mathrm{C}$.

MALDI-TOF mass spectrometry. The mass profile of AMOT peptide incubated in the presence or absence of JMJD6 enzyme (100 and $200 \mathrm{ng}$ ) was analyzed at the Analytical Facility for Bioactive Molecules at the Hospital for Sick Children (Toronto) using MALDI time-of-flight mass spectrometry (MALDI-TOF MS). Four microliters of enzyme reaction mixture was mixed with $6 \mu 1$ of $15 \mathrm{mg} / \mathrm{ml}$ 9-aminoacridine matrix in methanol (Sigma-Aldrich) and $1 \mu \mathrm{l}$ of this mixture was spotted on a MALDI plate, exposed to an Nd:YAG laser $(337 \mathrm{~nm})$ at 3-ns pulse width and $200-\mathrm{Hz}$ firing rate, and the mass of peptide was analyzed by timeof-flight tandem mass spectrometer (SCIEX TOF/TOFTM 5800 System; SCIEX) and TOF/TOF Series Explorer Software (SCIEX). Negative-ion reflector mode was used to acquire the spectra data derived from 200 individual laser shots from different positions on the spotted sample. Data Explorer Software (SCIEX) was used for processing of spectra.

Statistics. GraphPad Prism 7 software was used for statistical analysis. For the comparison of 2 groups with $n>3$, a nonparametric Mann-Whitney test was used. For the comparison of 2 groups with $n=3$, a 2-tailed unpaired Student's $t$ test was used to infer statistical significance. For the comparison of 3 or more groups, pairwise Mann-Whitney $U$ tests between individual groups were used. Data are presented as mean \pm SEM. All $P$ values were 2 tailed. Statistical significance was accepted at $P<0.05\left({ }^{*} P<0.05,{ }^{* *} P<0.01,{ }^{* *} P<0.001\right)$.

Study approval. The study was approved by the Mount Sinai Hospital Research Ethics Board (REB number 11-0287-E). Informed consent was given by all participants according to University of Toronto Faculty of Medicine Ethics guidelines, and the Research Ethics Board of Mount Sinai Hospital. 


\section{Author contributions}

$\mathrm{AF}$ and IC were responsible for concept and experimental design. AF performed the majority of experiments and analysis. AT conducted immunohistochemistry. SA conducted in vitro hydroxylation reactions, and contributed to loss/gain of JMJD6 studies and immunofluorescence of HTR-8/Svneo cells. LE conducted MALDI-TOF analysis. ML performed method development for the time-lapse imaging studies and assisted with data analysis. AF, IC, and MP wrote the manuscript.

\section{Acknowledgments}

Funding support was provided by Canadian Institutes of Health Research (CIHR) grant MOP 133436 (to IC). The authors thank the Research Centre for Women's and Infants' Health (RCWIH) Biobank for providing human tissue samples used in this study (http://biobank.lunenfeld.ca).

Address correspondence to: Isabella Caniggia, Mount Sinai Hospital, Lunenfeld-Tanenbaum Research Institute, 25 Orde Street, Room 6-1004-3, Toronto, Ontario, Canada M5T 3H7. Phone: 416.586.4803; Email: caniggia@lunenfeld.ca.

1. Troyanovsky B, Levchenko T, Månsson G, Matvijenko O, Holmgren L. Angiomotin: an angiostatin binding protein that regulates endothelial cell migration and tube formation. J Cell Biol. 2001;152(6):1247-1254.

2. Wells CD, et al. A Rich1/Amot complex regulates the Cdc42 GTPase and apical-polarity proteins in epithelial cells. Cell. 2006;125(3):535-548.

3. Ernkvist M, et al. The Amot/Patj/Syx signaling complex spatially controls RhoA GTPase activity in migrating endothelial cells. Blood. 2009;113(1):244-253.

4. Sugihara-Mizuno Y, et al. Molecular characterization of angiomotin/JEAP family proteins: interaction with MUPP1/Patj and their endogenous properties. Genes Cells. 2007;12(4):473-486.

5. Yi C, et al. A tight junction-associated Merlin-angiomotin complex mediates Merlin's regulation of mitogenic signaling and tumor suppressive functions. Cancer Cell. 2011;19(4):527-540.

6. Aase K, et al. Angiomotin regulates endothelial cell migration during embryonic angiogenesis. Genes Dev. 2007;21(16):2055-2068.

7. Leung CY, Zernicka-Goetz M. Angiomotin prevents pluripotent lineage differentiation in mouse embryos via Hippo pathway-dependent and -independent mechanisms. Nat Commun. 2013;4:2251.

8. Hirate Y, et al. Polarity-dependent distribution of angiomotin localizes Hippo signaling in preimplantation embryos. Curr Biol. 2013;23(13):1181-1194.

9. Soares MJ, Iqbal K, Kozai K. Hypoxia and placental development. Birth Defects Res. 2017;109(17):1309-1329.

10. Caniggia I, et al. Hypoxia-inducible factor-1 mediates the biological effects of oxygen on human trophoblast differentiation through TGFbeta(3). J Clin Invest. 2000;105(5):577-587.

11. Caniggia I, Winter JL. Adriana and Luisa Castellucci Award lecture 2001. Hypoxia inducible factor-1: oxygen regulation of trophoblast differentiation in normal and pre-eclamptic pregnancies--a review. Placenta. 2002;23 Suppl A:S47-S57.

12. American College of Obstetricians Gynecologists, Task Force on Hypertension in Pregnancy. Hypertension in pregnancy. Report of the American College of Obstetricians and Gynecologists' Task Force on Hypertension in Pregnancy. Obstet Gynecol. 2013;122(5):1122-1131.

13. Redline RW, Patterson P. Pre-eclampsia is associated with an excess of proliferative immature intermediate trophoblast. Hum Pathol. 1995;26(6):594-600.

14. Robertson WB, Brosens I, Landells WN. Abnormal placentation. Obstet Gynecol Annu. 1985;14:411-426.

15. Naicker T, Khedun SM, Moodley J, Pijnenborg R. Quantitative analysis of trophoblast invasion in preeclampsia. Acta Obstet Gynecol Scand. 2003;82(8):722-729.

16. Soleymanlou N, et al. Molecular evidence of placental hypoxia in preeclampsia. J Clin Endocrinol Metab. 2005;90(7):4299-4308.

17. Rolfo A, et al. Abnormalities in oxygen sensing define early and late onset preeclampsia as distinct pathologies. PLoS One. 2010;5(10):e13288.

18. Alahari S, Post M, Caniggia I. Jumonji domain containing protein 6: A novel oxygen sensor in the human placenta. Endocrinology. 2015;156(8):3012-3025.

19. Alahari S, Post M, Rolfo A, Weksberg R, Caniggia I. Compromised JMJD6 histone demethylase activity affects VHL gene repression in preeclampsia. J Clin Endocrinol Metab. 2018;103(4):1545-1557.

20. Wang F, et al. JMJD6 promotes colon carcinogenesis through negative regulation of p53 by hydroxylation. PLoS Biol. 2014;12(3):e1001819.

21. Unoki M, et al. Lysyl 5-hydroxylation, a novel histone modification, by Jumonji domain containing 6 (JMJD6). J Biol Chem. 2013;288(9):6053-6062.

22. Lee YF, et al. JMJD6 is a driver of cellular proliferation and motility and a marker of poor prognosis in breast cancer. Breast Cancer Res. 2012;14(3):R85.

23. Poulard C, et al. Role of JMJD6 in breast tumourigenesis. PLoS One. 2015;10(5):e0126181.

24. Ernkvist M, et al. p130-Angiomotin associates to actin and controls endothelial cell shape. FEBS J. 2006;273(9):2000-2011.

25. Ernkvist M, et al. Differential roles of p80- and p130-angiomotin in the switch between migration and stabilization of endothelial cells. Biochim Biophys Acta. 2008;1783(3):429-437.

26. Ietta F, et al. Dynamic HIF1A regulation during human placental development. Biol Reprod. 2006;75(1):112-121. 
27. Ray JE, Garcia J, Jurisicova A, Caniggia I. Mtd/Bok takes a swing: proapoptotic Mtd/Bok regulates trophoblast cell proliferation during human placental development and in preeclampsia. Cell Death Differ. 2010;17(5):846-859.

28. Xu J, et al. Aberrant TGF $\beta$ signaling contributes to altered trophoblast differentiation in preeclampsia. Endocrinology. 2016;157(2):883-899.

29. Caniggia I, Grisaru-Gravnosky S, Kuliszewsky M, Post M, Lye SJ. Inhibition of TGF-beta 3 restores the invasive capability of extravillous trophoblasts in preeclamptic pregnancies. J Clin Invest. 1999;103(12):1641-1650.

30. Wang W, Li N, Li X, Tran MK, Han X, Chen J. Tankyrase Inhibitors target YAP by stabilizing angiomotin family proteins. Cell Rep. 2015;13(3):524-532.

31. Bose R, Wrana JL. Regulation of Par6 by extracellular signals. Curr Opin Cell Biol. 2006;18(2):206-212.

32. Sivasubramaniyam T, et al. Where polarity meets fusion: role of Par6 in trophoblast differentiation during placental development and preeclampsia. Endocrinology. 2013;154(3):1296-1309.

33. Assémat E, Bazellières E, Pallesi-Pocachard E, Le Bivic A, Massey-Harroche D. Polarity complex proteins. Biochim Biophys Acta. 2008;1778(3):614-630.

34. Bratt A, et al. Angiomotin belongs to a novel protein family with conserved coiled-coil and PDZ binding domains. Gene 2002;298(1):69-77.

35. Ozdamar B, Bose R, Barrios-Rodiles M, Wang HR, Zhang Y, Wrana JL. Regulation of the polarity protein Par6 by TGFbeta receptors controls epithelial cell plasticity. Science. 2005;307(5715):1603-1609.

36. Wang HR, Ogunjimi AA, Zhang Y, Ozdamar B, Bose R, Wrana JL. Degradation of RhoA by Smurf1 ubiquitin ligase. Meth Enzymol. 2006;406:437-447.

37. Mantri M, Loik ND, Hamed RB, Claridge TD, McCullagh JS, Schofield CJ. The 2-oxoglutarate-dependent oxygenase JMJD6 catalyses oxidation of lysine residues to give 5S-hydroxylysine residues. Chembiochem. 2011;12(4):531-534.

38. Chang B, Chen Y, Zhao Y, Bruick RK. JMJD6 is a histone arginine demethylase. Science. 2007;318(5849):444-447.

39. Ismail HD, Newman RH, Kc DB. RF-Hydroxysite: a random forest based predictor for hydroxylation sites. Mol Biosyst. 2016;12(8):2427-2435

40. Wakeland AK, et al. Hypoxia directs human extravillous trophoblast differentiation in a hypoxia-inducible factor-dependent manner. Am J Pathol. 2017;187(4):767-780.

41. Genbacev O, Joslin R, Damsky CH, Polliotti BM, Fisher SJ. Hypoxia alters early gestation human cytotrophoblast differentiation/invasion in vitro and models the placental defects that occur in preeclampsia. J Clin Invest. 1996;97(2):540-550.

42. Levchenko T, Bratt A, Arbiser JL, Holmgren L. Angiomotin expression promotes hemangioendothelioma invasion. Oncogene. 2004;23(7):1469-1473

43. Hirate Y, Sasaki H. The role of angiomotin phosphorylation in the Hippo pathway during preimplantation mouse development Tissue Barriers. 2014;2(1):e28127.

44. Xu J, Lamouille S, Derynck R. TGF-beta-induced epithelial to mesenchymal transition. Cell Res. 2009;19(2):156-172.

45. Graham CH, Lysiak JJ, McCrae KR, Lala PK. Localization of transforming growth factor-beta at the human fetal-materna interface: role in trophoblast growth and differentiation. Biol Reprod. 1992;46(4):561-572.

46. Chan SW, Lim CJ, Chong YF, Pobbati AV, Huang C, Hong W. Hippo pathway-independent restriction of TAZ and YAP by angiomotin. J Biol Chem. 2011;286(9):7018-7026.

47. Zhao B, et al. Angiomotin is a novel Hippo pathway component that inhibits YAP oncoprotein. Genes Dev. 2011;25(1):51-63.

48. Bratt A, et al. Angiomotin regulates endothelial cell-cell junctions and cell motility. J Biol Chem. 2005;280(41):34859-34869.

49. Quiros M, Nusrat A. RhoGTPases, actomyosin signaling and regulation of the epithelial apical junctional complex. Semin Cell Dev Biol. 2014;36:194-203

50. James JL, et al. Isolation and characterisation of a novel trophoblast side-population from first trimester placentae. Reproduction 2015;150(5):449-462.

51. Okae H, et al. Derivation of human trophoblast stem cells. Cell Stem Cell. 2018;22(1):50-63.e6.

52. Heller B, et al. Amot recognizes a juxtanuclear endocytic recycling compartment via a novel lipid binding domain. J Biol Chem 2010;285(16):12308-12320

53. Graham CH, Connelly I, MacDougall JR, Kerbel RS, Stetler-Stevenson WG, Lala PK. Resistance of malignant trophoblast cells to both the anti-proliferative and anti-invasive effects of transforming growth factor-beta. Exp Cell Res. 1994;214(1):93-99.

54. Xu G, Chakraborty C, Lala PK. Expression of TGF-beta signaling genes in the normal, premalignant, and malignant human trophoblast: loss of smad3 in choriocarcinoma cells. Biochem Biophys Res Commun. 2001;287(1):47-55.

55. Levchenko T, Aase K, Troyanovsky B, Bratt A, Holmgren L. Loss of responsiveness to chemotactic factors by deletion of the C-terminal protein interaction site of angiomotin. J Cell Sci. 2003;116(Pt 18):3803-3810.

56. Moleirinho S, Guerrant W, Kissil JL. The Angiomotins--from discovery to function. FEBS Lett. 2014;588(16):2693-2703.

57. Dai X, et al. Phosphorylation of angiomotin by Lats $1 / 2$ kinases inhibits F-actin binding, cell migration, and angiogenesis. $J$ Biol Chem. 2013;288(47):34041-34051.

58. Lv M, et al. Angiomotin promotes renal epithelial and carcinoma cell proliferation by retaining the nuclear YAP. Oncotarget. 2016;7(11):12393-12403.

59. Lv M, et al. Angiomotin promotes breast cancer cell proliferation and invasion. Oncol Rep. 2015;33(4):1938-1946

60. DaSilva-Arnold S, James JL, A1-Khan A, Zamudio S, Illsley NP. Differentiation of first trimester cytotrophoblast to extravillous trophoblast involves an epithelial-mesenchymal transition. Placenta. 2015;36(12):1412-1418.

61. DaSilva-Arnold SC, et al. Human trophoblast epithelial-mesenchymal transition in abnormally invasive placenta. Biol Reprod. 2018;99(2):409-421.

62. Mondon F, et al. Profiling of oxygen-modulated gene expression in early human placenta by systematic sequencing of suppressive subtractive hybridization products. Physiol Genomics. 2005;22(1):99-107.

63. Mojallal M, et al. AmotL2 disrupts apical-basal cell polarity and promotes tumour invasion. Nat Commun. 2014;5:4557.

64. Wang H, et al. Tankyrase inhibitor sensitizes lung cancer cells to endothelial growth factor receptor (EGFR) inhibition via stabilizing angiomotins and inhibiting YAP signaling. J Biol Chem. 2016;291(29):15256-15266.

65. Rahman S, et al. The Brd4 extraterminal domain confers transcription activation independent of pTEFb by recruiting multiple 
proteins, including NSD3. Mol Cell Biol. 2011;31(13):2641-2652.

66. Sanjabi S, et al. A c-Rel subdomain responsible for enhanced DNA-binding affinity and selective gene activation. Genes Dev. 2005;19(18):2138-2151.

67. Kalkat M, et al. Placental autophagy regulation by the BOK-MCL1 rheostat. Autophagy. 2013;9(12):2140-2153 